\title{
On the excitation of the infrared knots along protostellar jets ${ }^{\star \star \star}$
}

\author{
T. Giannini ${ }^{1}$, C. McCoey ${ }^{2}$, A. Caratti o Garatti ${ }^{1,3}$, B. Nisini ${ }^{1}$, D. Lorenzetti ${ }^{1}$, and D. R. Flower ${ }^{2}$ \\ 1 INAF-Osservatorio Astronomico di Roma, via Frascati 33, 00040 Monteporzio Catone, Italy \\ 2 Physics Department, The University, Durham DH1 3LE, UK \\ 3 Università degli Studi “Tor Vergata”, via della Ricerca Scientifica 1, 00133 Roma, Italy
}

Received 16 July 2003 / Accepted 27 February 2004

\begin{abstract}
The complete near infrared $(0.9-2.5 \mu \mathrm{m})$ spectra in three different star forming regions (HH24-26, HH72 and BHR71) are presented and analyzed in the framework of shock excitation models. The spectra are dominated by $\mathrm{H}_{2}$ rovibrational emission (vibrational state $v \leq 5$, excitation energy $T_{\mathrm{ex}} \leq 35000 \mathrm{~K}$ ), while emission from ionized material, recognizable from [Fe II] and [S II] lines, is significantly fainter. The analysis of the $\mathrm{H}_{2}$ excitation diagrams points to the existence of two different excitation regimes: whilst condensations observed only in the infrared appear to have temperatures rarely exceeding $3000 \mathrm{~K}$ and can be modelled in the framework of steady-state C-shock models, the infrared counterparts of Herbig Haro (HH) objects exhibit a temperature stratification with components up to more than $5000 \mathrm{~K}$. The $\mathrm{H}_{2}$ emission from representative HH objects (HH26A, HH72A and HH320A) has been successfully modelled by planar J-shocks with magnetic precursors, for which the main parameters (pre-shock density, speed) are derived. However, these same models are unable to reproduce the observed atomic and ionic emission, which probably arises from a distinct and perhaps more embedded region with respect to that traced by the $\mathrm{H}_{2}$. Some of the physical parameters of such regions (fractional ionization, density) have been estimated in $\mathrm{HH} 72$, on the basis of the observed ionic lines.
\end{abstract}

Key words. stars: circumstellar matter - infrared: ISM - ISM: Herbig-Haro objects - ISM: jets and outflows - shock waves

\section{Introduction}

The physical effect exerted on the ambient medium by the impact of jets from accreting protostars results in the formation of shock waves. The compressed and heated gas radiates away the accumulated thermal energy through the emission of ionic, atomic and molecular lines, in relative proportions which depend on the structure of the shock wave. During the gas cooling, a fundamental role is played by molecular hydrogen, whose high abundance compensates the low transition rates due to its homonuclear nature. In the near infrared, the association of $\mathrm{H}_{2}$ emission with knots of shocked gas has been demonstrated by mapping at sub-arcsecond scale in the $v=1-0 \mathrm{~S}(1)$ line at $2.122 \mu \mathrm{m}$ (e.g. Eislöffel 2000), and the rovibrational lines are effective probes of the molecular gas at thousands of kelvin, which is missed by optical observations. $\mathrm{H}_{2}$ line intensities and intensity ratios are also extensively used to distinguish between fluorescence and shock excitation as possible mechanisms at the origin of the emission, since strong $\mathrm{H}_{2}$ lines with $v \geq 6$ in the $1.0-1.4 \mu \mathrm{m}$ range are

Send offprint requests to: T. Giannini,

e-mail: teresa@coma.mporzio.astro.it

* Based on observations collected at the European Southern Observatory, La Silla, Chile (66.C-158, 68.C-0020, 69.C-0175).

$\star \star$ Tables 1-3 are only available in electronic form at: http://www.edpsciences.org expected if fluorescence is responsible for the emission (Black \& van Dishoeck 1987). It has proved more difficult to derive the nature (C-ontinuous or J-ump type, e.g. Kaufman \& Neufeld 1996; Hollenbach \& McKee 1989; Draine 1980) and the physical parameters of the shock waves, such as the velocity, the preshock density, the strength of the transverse magnetic field and the temperature of the neutral gas. In the conventional scenario, C-type shocks can exist up to velocities typically $\approx 50 \mathrm{~km} \mathrm{~s}^{-1}$, beyond which the $\mathrm{H}_{2}$ molecule is collisionally dissociated and the kinetic temperature of the gas rapidly increases, giving rise to a discontinuity in the shock parameters. However, more recent models (e.g. Smith 1995; Le Bourlot et al. 2002; Flower et al. 2003) have shown that $\mathrm{H}_{2}$ dissociation can be inhibited over a wider range of shock parameters: from slow $\left(v_{\mathrm{s}} \lesssim 25 \mathrm{~km} \mathrm{~s}^{-1}\right.$ ), partially dissociative J-type shocks, up to fast C-type shocks travelling at 70$80 \mathrm{~km} \mathrm{~s}^{-1}$. From an observational point of view, the presence of different shock components (J- or C-type) can be demonstrated by the modifications induced in the $\mathrm{H}_{2}$ excitation diagram: the lower post-shock densities attained in Cshocks result in a larger departure from LTE conditions than in J-shocks of the same speed (Flower et al. 2003). In order to probe such deviations, it is essential to investigate spectroscopically the 1.0-1.4 $\mu \mathrm{m}$ range, since several $\mathrm{H}_{2}$ lines with different vibrational quantum number and of relatively high excitation energy $(>15000 \mathrm{~K})$ lie at these wavelengths. 
With the aim of observing such lines, we have undertaken a spectroscopic survey (from 0.9 to $2.5 \mu \mathrm{m}$ ) of a sample of Herbig-Haro $(\mathrm{HH})$ objects and $\mathrm{H}_{2}$ jets, based on the observations gathered with the SOFI spectrometer at the ESO-NTT. We have already reported the results of this survey regarding HH43, HH111, HH240/241 and HH120 (Giannini et al. 2002; Nisini et al. 2002, hereafter Papers I and II, respectively), showing that quite different excitation conditions can occur in $\mathrm{HH}$ objects: while in $\mathrm{HH} 43$ the bulk of the cooling occurs in $\mathrm{H}_{2}$ lines, the spectra of the other three objects are dominated by iron lines. In $\mathrm{HH} 43$, the $\mathrm{H}_{2}$ emission has been successfully fitted by means of high velocity, C-type shock models (Flower et al. 2003), whereas, in the other three cases, the strong ionic emission testifies in favour of a dissociative component in the shock structure. Here we present the observations obtained in three other regions, namely HH24-26, HH72 and $\mathrm{HH} 320 / 321$ (BHR71). All the spectra exhibit copious $\mathrm{H}_{2}$ rovibrational emission, together, in some cases, with a fainter atomic and ionic component. Therefore, they represent valid tests of the ability of current shock models to predict simultaneously molecular and atomic/ionic emission and an opportunity to check the validity of the underlying assumptions of the models.

The structure of the paper is the following: we present the targetted regions in Sect. 2 and then describe the observations and the results obtained (Sect. 3). In Sect. 4, we derive the physical parameters of the emitting gas and model the observed emission, assuming shock excitation. Section 5 summarizes our conclusions.

\section{The investigated regions}

\subsection{The HH24-26 region}

Located in the L1630 Orion dark cloud ( $d \approx 400$ pc, AnthonyTwarog 1982), the HH24-26 region is an active site of star formation, rich in young stellar objects in different evolutionary stages. Two low luminosity, Class 0 protostars (André et al. 2000), HH24MMS (Chini et al. 1993, $\alpha_{2000}=05^{\mathrm{h}} 46^{\mathrm{m}} 06.7^{\mathrm{s}}$, $\left.\delta_{2000}=-00^{\circ} 10^{\prime} 40.8^{\prime \prime}, l^{\mathrm{II}}=205.49, b^{\mathrm{II}}=-14.57, L_{\mathrm{bol}}=5 L_{\odot}\right)$ and HH25MMS (Bontemps et al. 1995, $\alpha_{2000}=05^{\mathrm{h}} 46^{\mathrm{m}} 06.7^{\mathrm{s}}$, $\left.\delta_{2000}=-00^{\circ} 13^{\prime} 24.7^{\prime \prime}, l^{\mathrm{II}}=205.53, b^{\mathrm{II}}=-14.60, L_{\mathrm{bol}}=6 L_{\odot}\right)$ drive distinct compact jets, traced by means of both the $2.12 \mu \mathrm{m}$ $\mathrm{H}_{2}$ line and CO mm transitions (Gibb \& Davis 1998; Bontemps et al. 1996), while the Class I protostar HH26IR (Davis et al. $1997, \alpha_{2000}=05^{\mathrm{h}} 46^{\mathrm{m}} 03.9^{\mathrm{s}}, \delta_{2000}=-00^{\circ} 14^{\prime} 52.5^{\prime \prime}$, $\left.l^{\mathrm{II}}=205.54, b^{\mathrm{II}}=-14.62, L_{\mathrm{bol}}=28.8 L_{\odot}\right)$ gives rise to a more extended molecular outflow (Gibb \& Heaton 1993). Imaging in the $2.12 \mu \mathrm{m} \mathrm{H}_{2}$ rovibrational line (Davis et al. 1997, see Fig. 1) reveals a string of shocked $\mathrm{H}_{2}$ knots along the axis of the HH25MMS and HH26IR outflows. Herbig-Haro objects ${ }^{1}$ (HH24, HH25 and HH26) are associated with each of these three sources. Proper motions were measured in HH25 and 26 by Chrysostomou et al. (2000), who found velocities ranging from less than 70 up to $200 \mathrm{~km} \mathrm{~s}^{-1}$; the lowest value pertains to HH26A, which should be a stationary shock region, and the

\footnotetext{
${ }^{1}$ In the following we will refer to $\mathrm{HH}$ objects as the optically visible condensations.
}

largest one to HH26C in the working surface of a bow shock. Far infrared spectra of the region were obtained by Benedettini et al. (2000), who interpreted the observed emission (from [O I], $\mathrm{CO}, \mathrm{H}_{2} \mathrm{O}$ and $\mathrm{H}_{2}$ lines) as due to a mixture of both $\mathrm{C}$ - and J-type shocks. Recently, Fabry-Perot observations have demonstrated the existence of elongated $\mathrm{H}_{2}$ emission, on scales of a few arcsec, from HH26IR and HH25MMS, which appears associated with the base of the larger scale jets (Davis et al. 2002).

\subsection{The HH72 region (L1660)}

The $\mathrm{L} 1660$ region is a bright-rimmed core illuminated by the young OB cluster NGC 2362 in Vela (Reipurth \& Graham 1988 ), at a distance of $\approx 1500 \mathrm{pc}$ (Hilton \& Lahulla 1995). An E-W, poorly collimated outflow (Schwartz et al. 1988) is present in the region, possibly driven by the intermediate luminosity IRAS source 07180-2356 (Reipurth et al. 1993, $\alpha_{2000}=$ $07^{\mathrm{h}} 20^{\mathrm{m}} 10.3^{\mathrm{s}}, \delta_{2000}=-24^{\circ} 02^{\prime} 24^{\prime \prime}, l^{\mathrm{II}}=237.52, b^{\mathrm{II}}=-4.83$, $\left.L_{\mathrm{bol}} \approx 170 L_{\odot}\right)$. Figure 2 shows the SOFI $2.12 \mu \mathrm{m}$ image of the region, where, together with the chain of $\mathrm{HH}$ objects (HH72 A-C, Reipurth \& Graham 1988), several pure $\mathrm{H}_{2}$ features are recognizable (HH72 D-Z). A jet of a few arcseconds has been recently observed close by the infrared source HH72IRS (Davis et al. 2002, see Fig. 2).

\subsection{The BHR71 region}

Figure 3 shows the $\mathrm{H}_{2} 2.12 \mu \mathrm{m}$ image by Bourke (2001) of the Bok globule BHR71 (Bourke et al. 1995), located at $\approx 200$ pc (Seidensticker \& Schmidt-Kaler 1989). An embedded binary protostellar system, separated by $\approx 3400 \mathrm{AU}$, has been found in the region (Bourke 2001). One of the two sources has been identified as a Class 0 protostar (IRS 1: $\alpha_{2000}=12^{\mathrm{h}} 01^{\mathrm{m}} 36.6^{\mathrm{s}}$, $\left.\delta_{2000}=-65^{\circ} 08^{\prime} 48.2^{\prime \prime}, l^{\mathrm{II}}=297.73, b^{\mathrm{II}}=-2.78, L_{\mathrm{bol}} \approx 9 L_{\odot}\right)$ by means of $1.3 \mathrm{~mm}$ observations (Bourke et al. 1997); ISO mid-IR maps led to the discovery of the possibly more evolved source IRS 2 (Bourke 2001, $\alpha_{2000}=12^{\mathrm{h}} 01^{\mathrm{m}} 34.0^{\mathrm{s}}, \delta_{2000}=$ $\left.-65^{\circ} 08^{\prime} 44.3^{\prime \prime}, l^{\mathrm{II}}=297.72, b^{\mathrm{II}}=-2.78\right)$. A recent study by Lemaire et al. (2002) suggests the presence of a circumstellar disk around this object. A large scale CO outflow, where a shock enhanced chemistry has been revealed (Garay et al. 1998), is driven by IRS1 along the North-South direction, while IRS2 gives rise to a more compact outflow with an inclination $\approx-36^{\circ}$. Optical [S II] images revealed $\mathrm{HH}$ associations (HH320 and HH321) along both the CO outflows (Corporon \& Reipurth 1997); these should be at a very low degree of excitation, as demonstrated by the lack of emission in the $\mathrm{H} \alpha$ line. Faint $\mathrm{H}_{2}$ emission features were found by Bourke (2001).

\section{Observations and results}

The observations were carried out during two runs (January 2001, 2002) with SOFI (Lidman \& Cuby 1999) at ESO-NTT $3.5 \mathrm{~m}$ telescope (La Silla). Long slit spectroscopy was obtained through the blue $(0.95-1.64 \mu \mathrm{m})$ and the red $(1.53-2.52 \mu \mathrm{m})$ grisms in the low resolution mode $(\lambda / \Delta \lambda \approx 600$ with the $1 \times 290$ arcsec slit). To cover most of the $\mathrm{H}_{2}$ emission knots present in the HH24-26 region we used five different slit orientations, which are depicted in Fig. 1. In the HH72 region, we 


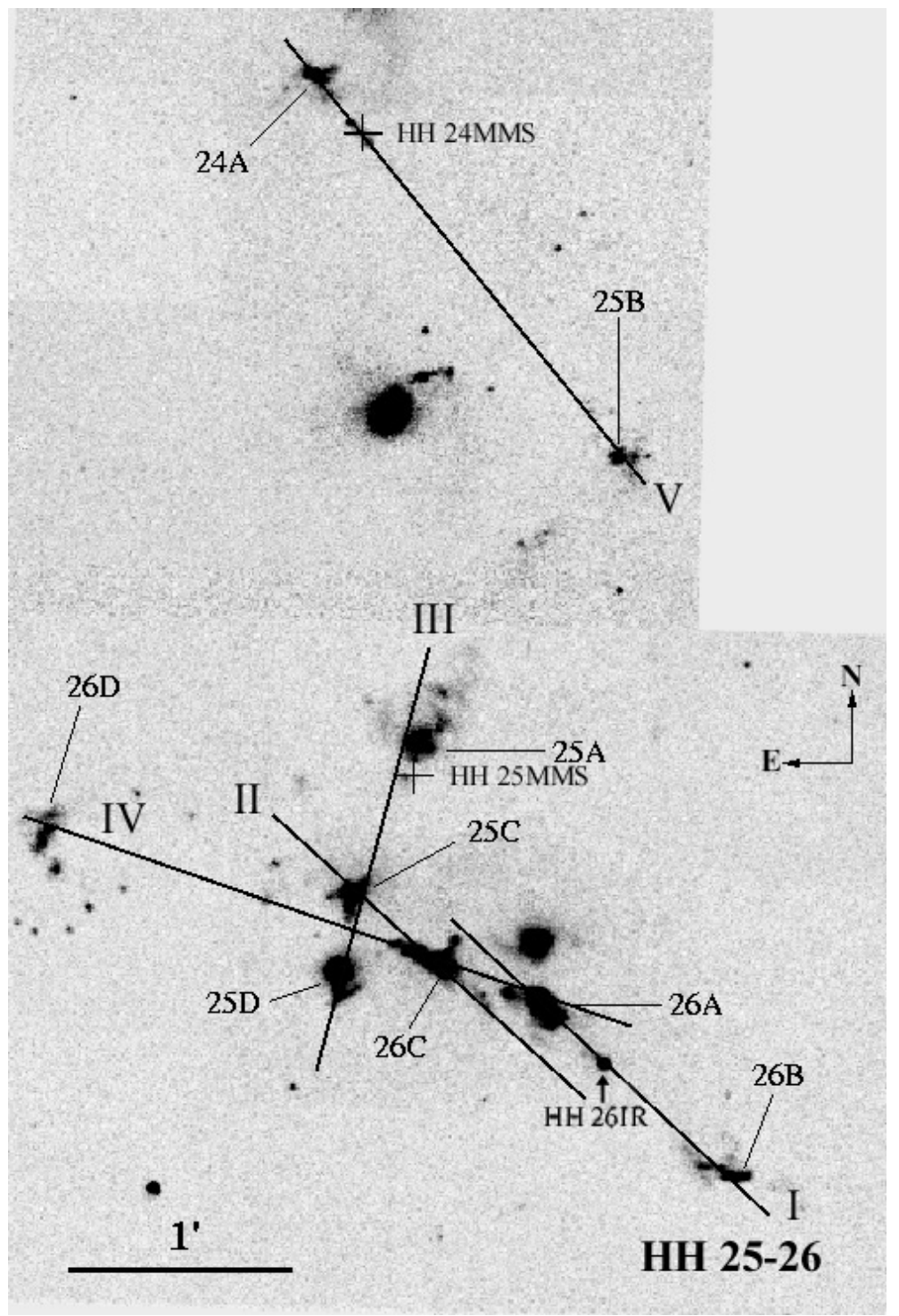

Fig. 1. Positions of the SOFI slit (I to V) in the HH24-26 region superimposed on the $\mathrm{H}_{2} 2.12 \mu \mathrm{m}$ image by Davis et al. (1997). The adopted PA (measured from north to east) are: $50^{\circ}$ (slits I, II); $164^{\circ}$ (slit III); $74^{\circ}$ (slit IV); $40^{\circ}$ (slit V). The outflows' exciting sources HH24MMS, HH25MMS and HH26IR are indicated.

adopted four slits to observe the knots both in the blueshifted (knots L-B) and redshifted (knots Y and Z) lobes of the outflow (Fig. 2), while the two jets present in BHR71 were investigated with three slits (see Fig. 3), covering both the Herbig-Haro objects $320 / 321$ and the fainter $\mathrm{H}_{2}$ emission features recognizable in the $2.12 \mu \mathrm{m}$ image by Bourke (2001). The observations were performed by nodding and jittering the telescope, keeping the target along the slit in the usual $\mathrm{ABB}$ ' $\mathrm{A}$ ' mode, with a total integration time of $1200 \mathrm{~s}$. The data reduction and calibration were performed by using the IRAF package. Each observation was flat fielded and sky subtracted, while to remove the atmospheric spectral response a couple of telluric O-type stars were observed before and after each on-source observation. Each target spectrum was then divided by the telluric spectrum, corrected by the blackbody function at the temperature of the star, and by a few intrinsic hydrogen absorption lines. Wavelength calibration was obtained from the spectrum of a Xenon-Argon lamp; the estimated wavelength error is smaller than the spectral resolution element ( 2 pixels $\approx 20 \AA$ ), which corresponds to a velocity of about $300 \mathrm{~km} \mathrm{~s}^{-1}$. No evidence of shifts larger than this limit has been found. Flux calibration (associated uncertainty 10\%) was obtained from the observation of some photometric standard stars from the Carter \& Meadow (1995) catalogue. Ratios between lines lying in the blue and red parts of the spectrum, are affected by a $10 \%$ uncertainty, due to the different instrumental response in the two segments. Such error has been estimated by using a line (e.g. [Fe II] $1.64 \mu \mathrm{m}$ ) present in both parts. As an example of our observations we show in Figs. 4-6 the spectra of HH26A, HH72A and HH320A, which are among the knots richest in lines; they are all dominated by $\mathrm{H}_{2}$ emission in form of rovibrational lines coming from levels with vibrational quantum number up to 5. A relatively faint atomic and ionic emission is also observed: in addition to the bright [CI] doublet at $0.98 \mu \mathrm{m}$, a few lines of [Fe II], [S II], and [N I] were detected in the most prominent knots. Tables 1a-c, 


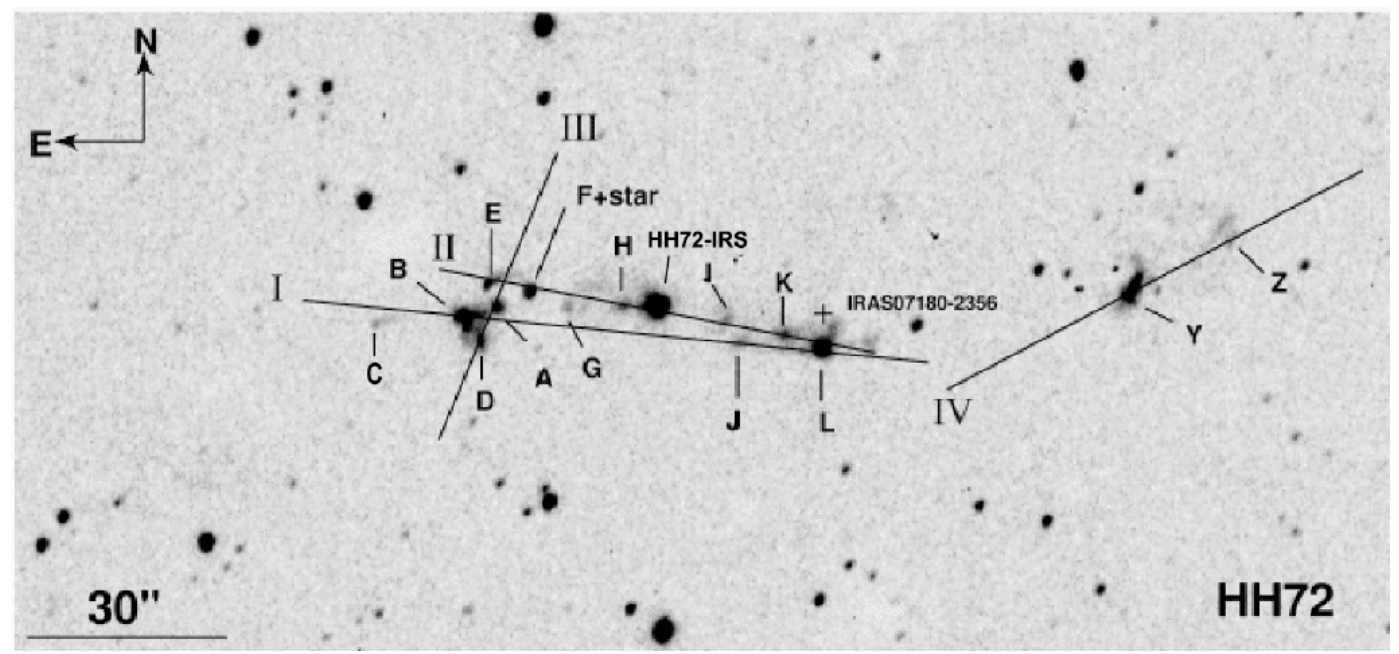

Fig. 2. Positions of the SOFI slit (I to IV) in the L1660 region (HH72) superimposed on the $\mathrm{H}_{2} 2.12 \mu$ m image taken at the NTT telescope. The adopted PA are: $84^{\circ}$ (slit I); $79^{\circ}$ (slit II); $157^{\circ}$ (slit III); $117^{\circ}$ (slit IV). The candidate jet's exciting source IRAS $07180-2356$ is indicated by a cross.

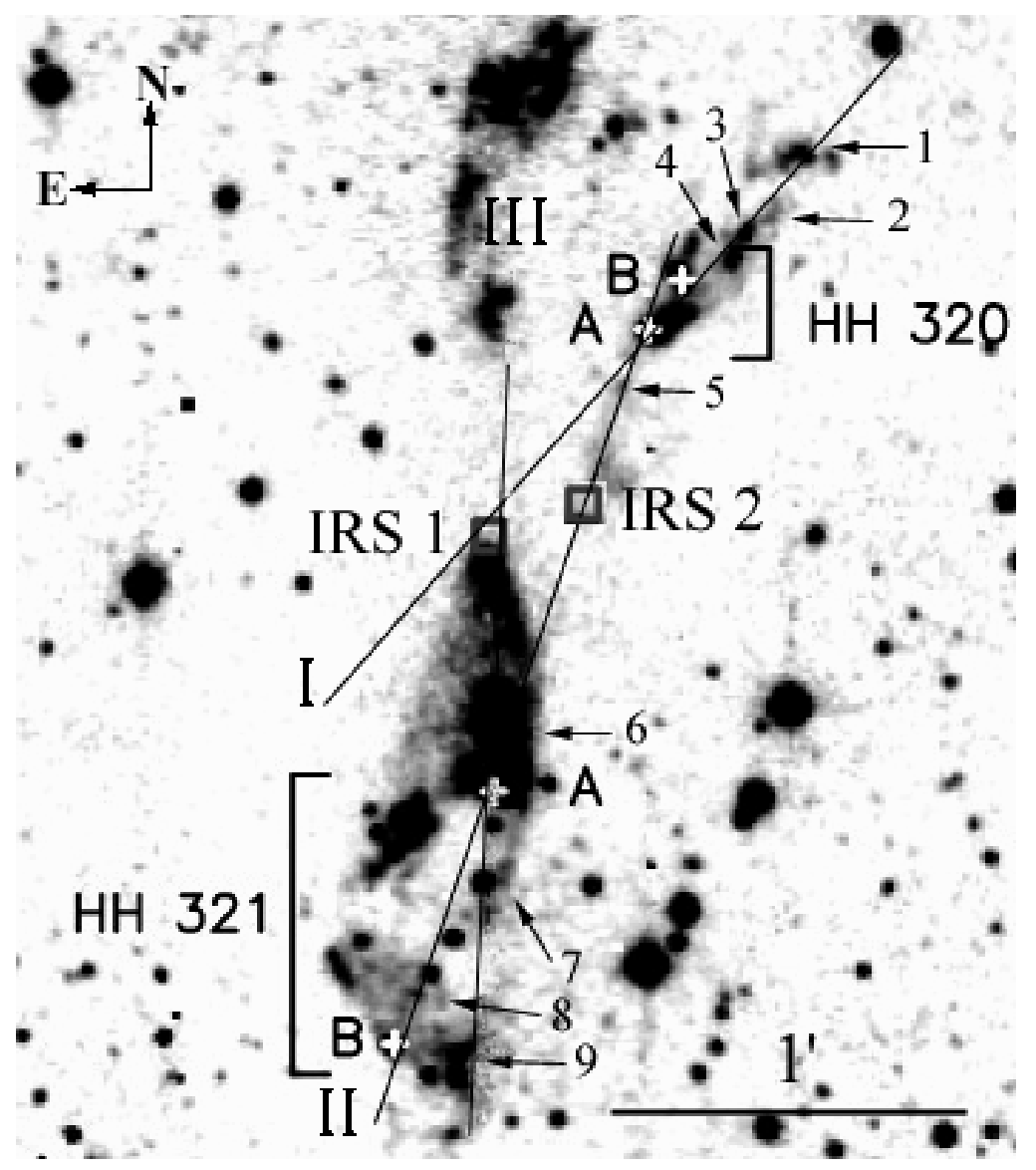

Fig. 3. Positions of the SOFI slit (from I to III) in BHR71 superimposed on the $\mathrm{H}_{2} 2.12 \mu \mathrm{m}$ image by Bourke (2001). The adopted PA are: $320^{\circ}$ (slit I); $339^{\circ}$ (slit II); $177^{\circ}$ (slit III). The two driving sources IRS1 and IRS2 (Bourke 2001) are indicated by rectangles. Together with the HH objects 320A/B and 321A/B, we label from 1 to 9 the pure $\mathrm{H}_{2}$ emission knots identified by Bourke (2001).

$2 \mathrm{a}-\mathrm{b}$ and $3 \mathrm{a}-\mathrm{b}$ (which are available only in the electronic version of the paper), list all the detected lines, along with their spectral identification and the vacuum wavelength. Some knots (e.g. HH26A, C) were intersected by two slits, but we did not find a significant variation in the line ratios along the different directions, although the emission is generally brighter along the jet axis. To the latter correspond the fluxes reported in the Tables. The integrated fluxes were obtained by fitting the line shape with a single (or double in case of blending) Gaussian profile. The reported uncertainty derives from the rms of the 
HH26 A
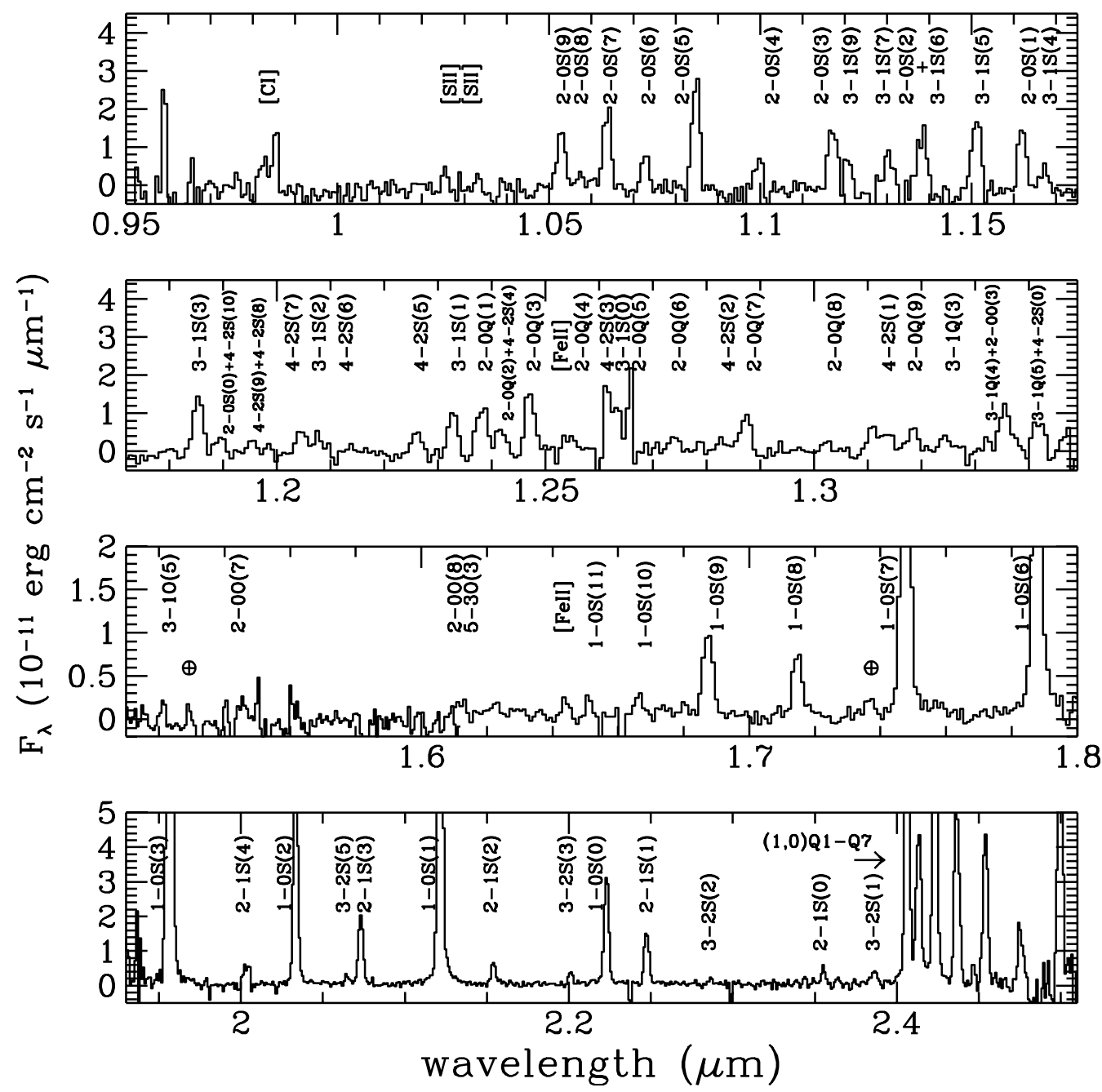

Fig. 4. Spectrum between 0.95 and $2.5 \mu \mathrm{m}$ of HH26A.

baseline, multiplied by the width of the instrumental profile (30 ̊).

The $\mathrm{H}_{2}$ 1-0 S(1) $2.12 \mu$ m line fluxes observed in HH24-26 and in $\mathrm{HH} 72$ are systematically lower, by a factor between 1.5 and 10, than the values derived from imaging by Davis et al. (1997). The discrepancies are attributable to the spatial extent of the objects observed, relative to our $1^{\prime \prime}$ slit width; this explanation is supported by the fact that the largest differences occur in those knots, such as HH26D, that we observed along their shortest axis (see Fig. 1).

From inspection of Tables 1a-1c, we see that, along the $\mathrm{HH} 26$ flow, copious $\mathrm{H}_{2}$ emission is detected only close to the driving source (HH26A and C), while in all the other knots faint and weakly excited $\mathrm{H}_{2}$ lines are revealed. The opposite situation exists along the HH72 jet (Tables $2 \mathrm{a}-2 \mathrm{~b}$ ), where the richest $\mathrm{H}_{2}$ spectra are emitted by the knots at the apex of the blueshifted lobe (HH72A and B). Only $\mathrm{H}_{2}$ emission has been detected toward the BHR71 jets (Tables 3a-3b). Particularly remarkable is the lack of the [CI] doublet, which, in addition to the present observations, has been detected in all the $\mathrm{HH}$ objects previously investigated (Papers I and II). We also note that, compared with the other Herbig-Haro objects in BHR71, HH321B has a significantly weaker spectrum, probably because the emission peak was badly covered by the slit.

To complement the SOFI observations, we obtained in April 2002 the 3-5 $\mu \mathrm{m}$ spectra of the HH72 and BHR71 regions with ISAAC at the VLT (Cuby et al. 2003). In this spectral range, several pure rotational lines of $\mathrm{H}_{2}$ with high excitation energy (up to $\approx 40000 \mathrm{~K}$ ) are located, which can be used to determine the density of the molecular gas (Giannini et al. 2001). Unfortunately, the poor atmospheric conditions prevented us to obtain high quality data at wavelengths larger than $4 \mu \mathrm{m}$, where both the thermal emission and the presence of strong water absorption bands critically affect the reliability of the observations. We used the $1 \times 120 \operatorname{arcsec} \operatorname{sit}(\lambda / \Delta \lambda \approx 2000)$, pointing at the Herbig-Haro objects $72 \mathrm{~A} / \mathrm{B}\left(\mathrm{PA}=84^{\circ}\right.$, i.e. slit I in Fig. 2$)$ and 320A/B (PA $=320^{\circ}$, i.e. slit I in Fig. 3). The observational strategy and the procedure adopted for the data reduction are similar to those reported by Giannini et al. (2001). We detected two $\mathrm{H}_{2}$ lines: the $1-0 \mathrm{O}(5)$ at $3.235 \mu \mathrm{m}$ in $\mathrm{HH} 72 \mathrm{~B}$ and the 

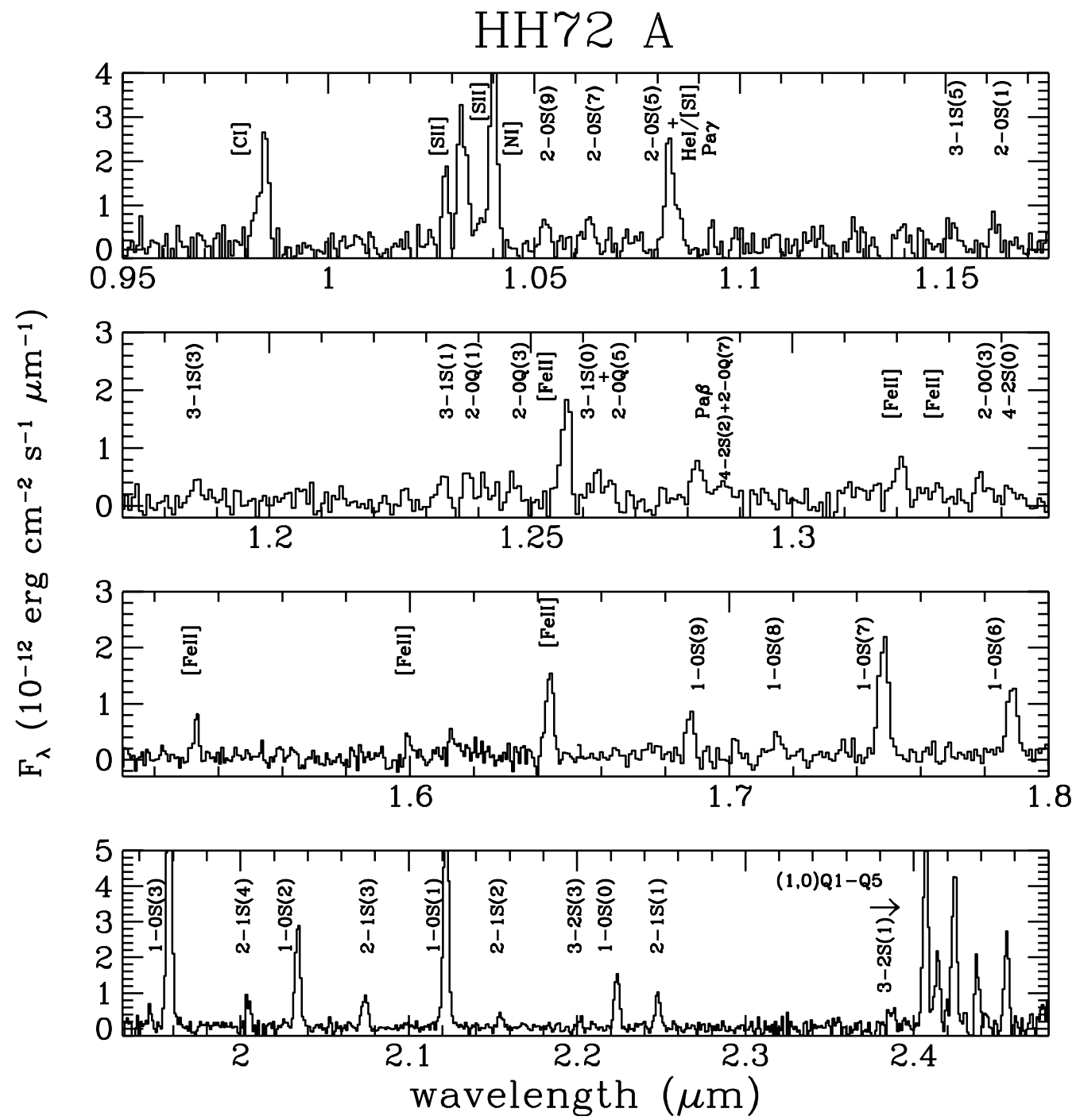

Fig. 5. Spectrum between 0.95 and $2.5 \mu \mathrm{m}$ of HH72A.

1-0 S(13) at 3.847 $\mu \mathrm{m}$ in HH72B and HH320A (Tables 2a, 3a). The extracted spectra are shown in Fig. 7. The 1-0 O(5) line appears narrower of about factor of 2 than the instrumental profile; however, since its trace in the bidimensional image of the spectrum is clearly recognizable, we consider the line to be a genuine detection. The estimated fluxes were used in conjunction with the SOFI data. No intercalibration correction was applied to the ISAAC and SOFI lines, but the consistency of these two data sets is supported by the fact that the column densities of lines coming from the same upper level are equal to within the error bars (see Sect. 4.2).

\section{Analysis and discussion}

\section{1. $A_{V}$ determination}

The line lists, reported in Tables $1-3$, clearly suggest that some differences in the $\mathrm{H}_{2}$ emission exist throughout all the observed knots. In particular, lines coming from the highest excited levels $(v \geq 3)$ are observed mainly in condensations visible also in the optical (e.g. HH26A/C, HH72A/B, HH320/321), while low excitation conditions are recognizable, with few exceptions, in the other knots (e.g. HH25, HH72E-Z). Since the most excited lines are located mainly in the blue part of the spectrum (i.e. at wavelengths shorter than $1.4 \mu \mathrm{m}$ ), their absence in the pure infrared knots could be interpreted as being due to a higher visual extinction, without necessarily implying lower excitation conditions. To investigate this crucial point, we have firstly estimated the visual extinction in the various knots by using pairs of transitions coming from the same upper level, for which the difference between the observed and the theoretical flux ratio is a function only of $A_{\mathrm{V}}$ (cf. Gredel 1994). The accuracy of this method relies strongly on the presence of pairs of bright lines at significantly different wavelengths which are not affected severely by observational problems. In practice, sufficient numbers of such pairs of lines are present in the spectra of only the brightest, optical knots. For this reason, the values of $A_{\mathrm{V}}$ derived in the brightest objects were applied also to the nearby knots, where insufficient lines are present to allow a specific determination. Given the large number of molecular hydrogen lines, the criteria cited above are satisfied by several 
HH320 A
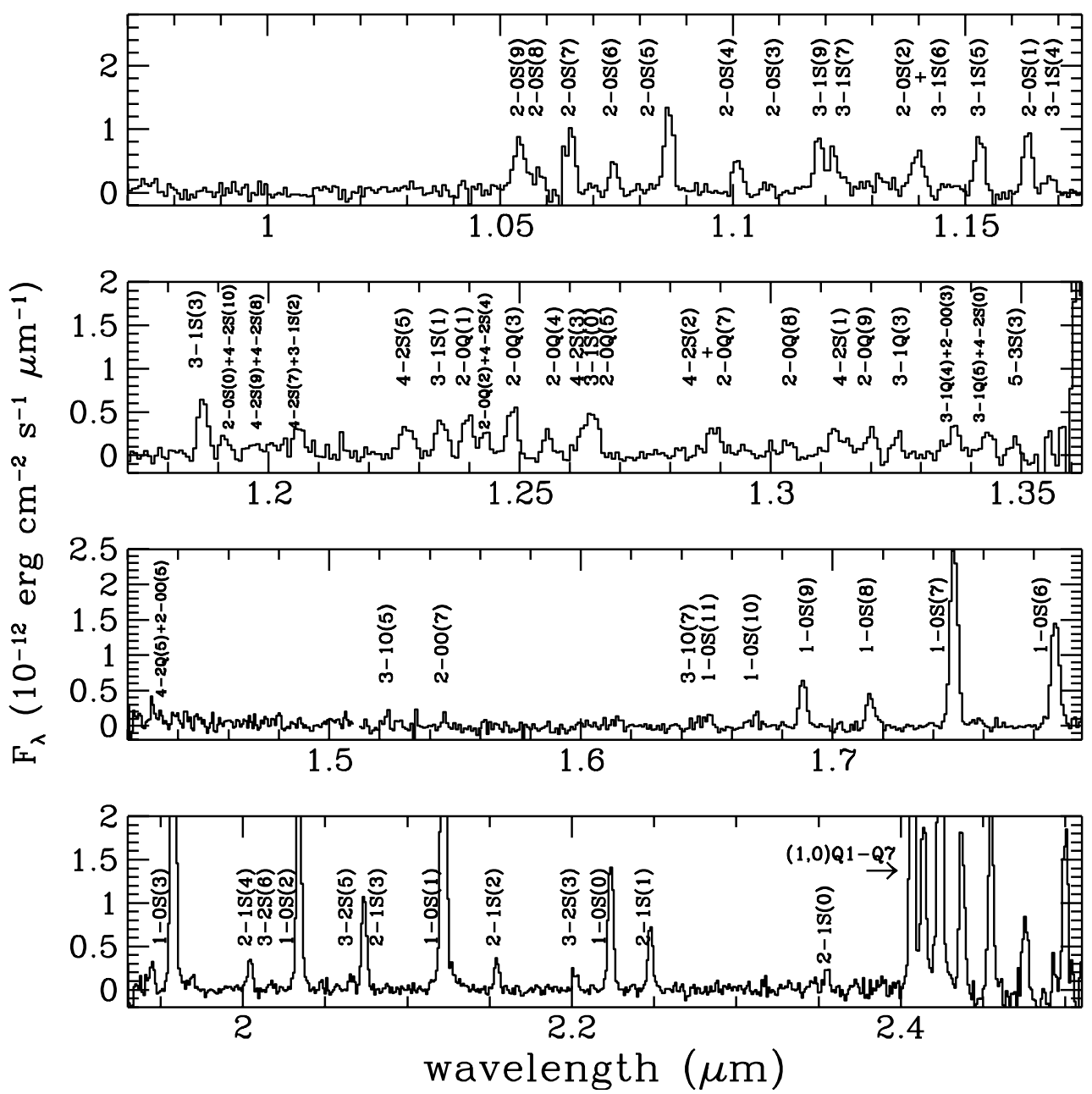

Fig. 6. Spectrum between 0.95 and $2.5 \mu \mathrm{m}$ of HH320A.

pairs of lines coming from the same upper level (e.g. of the kind 2-0 S(i)/2-0 Q(i+2) or 3-1 S(i)/3-2 S(i)). By using all the available ratios, we were able to estimate the visual extinction with an uncertainty of 1-2 mag. Adopting the reddening law of Rieke \& Lebofsky (1985), we found $A_{\mathrm{V}}$ up to 8 mag in the HH24-26 and HH72 flows, while the visual extinction in the BHR71 region is small ( $\left.A_{\mathrm{V}} \leq 2 \mathrm{mag}\right)$, however well compatible with the $A_{\mathrm{V}}$ values ( $<3 \mathrm{mag}$ ) estimated towards the galactic plane direction (e.g. Hakkila et al. 1997). The values of $A_{\mathrm{V}}$ for individual sources are given in Figs. 8-13. These results indicate that the differential extinction only marginally biases the observations in all the knots except those having $A_{\mathrm{V}}=8 \mathrm{mag}$ (HH25B, HH72J-Z). Thus the presence or absence of highly excited $\mathrm{H}_{2}$ lines in the spectra reflects in almost all cases the excitation conditions of the molecular gas. In this respect, the fact that we detect no lines with $v \geq 6$ indicates that fluorescence mechanisms do not play an important role in the excitation (Black \& van Dishoeck 1987).

An independent determination of $A_{\mathrm{V}}$ could be obtained from the [Fe II] lines at 1.257 and $1.644 \mu \mathrm{m}$. However, in our objects, these lines are generally detected with a poor signalto-noise ratio, with the only exception being HH72A. For this object, we derive $A_{\mathrm{V}[\mathrm{Fe} \text { II }]}=4.7 \pm 1.5 \mathrm{mag}$, a value higher than the negligible extinction indicated for this object by the $\mathrm{H}_{2}$ lines. The possible implications of this result will be discussed below.

\section{2. $\mathrm{H}_{2}$ emission}

In order to derive an estimate of the temperature along the flows, we have used the excitation diagrams (Boltzmann plots) for the $\mathrm{H}_{2}$ lines (Figs. 8-13), plotting the extinction-corrected column densities of the rovibrational levels, divided by the statistical weights, against their excitation energies. In each plot, lines coming from different vibrational states (from $v=0$ to $v=5$ ) are depicted by different symbols. To minimize the uncertainties, we considered only unblended lines with a signalto-noise ratio greater than 3. Any significant deviation of the ortho:para ratio from its statistical value of 3 would be apparent as a misalignment of the ortho and para data points in the rotation diagrams. No such deviations are observed: see below and Figs. 8-13.

If the gas is excited at a single temperature, a straight line can be fitted through the data points: this seems to be 


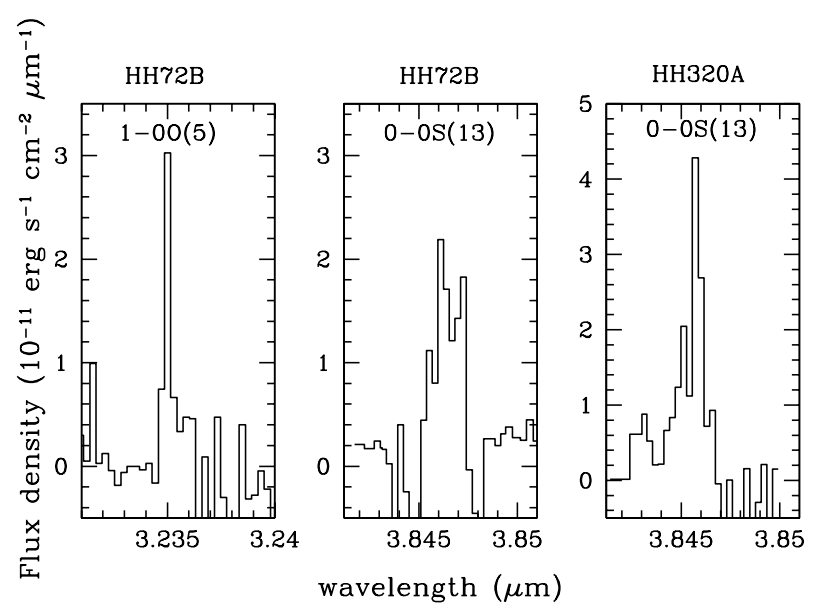

Fig. 7. $\mathrm{H}_{2}$ lines observed with ISAAC in HH72B and HH320A.

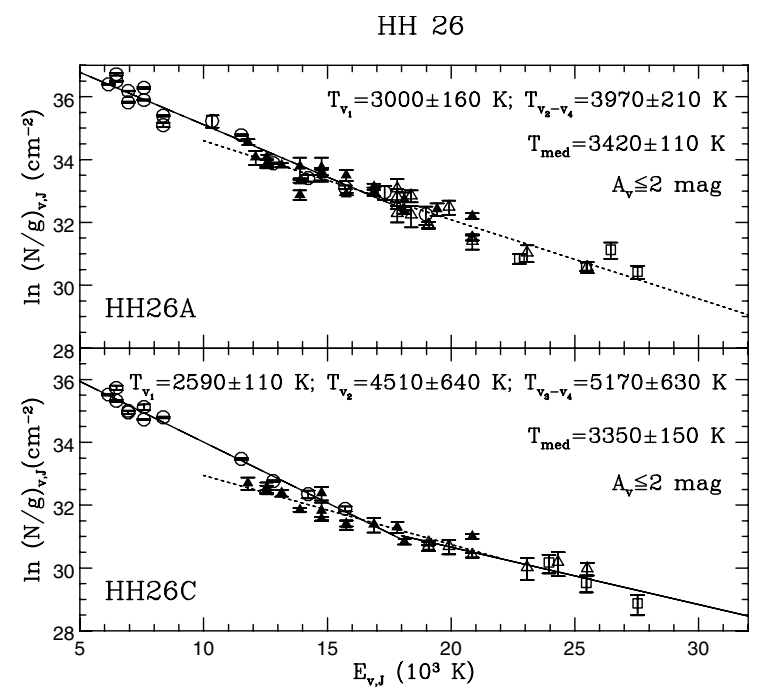

Fig. 8. Excitation diagram for $\mathrm{HH} 26 \mathrm{~A}$ and $\mathrm{B}$. Lines coming from different vibrational levels are indicated with different symbols: filled circles, open circles, filled triangles, open triangles, filled squares and open squares indicate lines coming from levels from 0 to 5 , respectively. The straight line(s) represents the best fit(s) through the data, at the temperature(s) reported in top right angle. The derived value of the extinction is also indicated.

the case only where lines with excitation energies lower than typically $15000 \mathrm{~K}$ are detected, i.e. in all the molecular hydrogen condensations and few Herbig-Haro objects (such as HH321B and HH25A). Here a single temperature typically between 2000 and $3000 \mathrm{~K}$ can account for the observed excitation diagrams.

Other Herbig-Haro objects (HH26A, HH72A/B, $\mathrm{HH} 320 \mathrm{~A} / \mathrm{B}$ and HH321A) and HH26C, which show a richer $\mathrm{H}_{2}$ spectrum, deserve a more realistic approach, which consists of relaxing the assumption of a single excitation temperature and fitting the various vibrational series separately. Although the average temperature remains quite similar to that measured in the molecular condensations, deviations from the thermal equilibrium are evident, with a spread from $2000 \mathrm{~K}$ to more than $5000 \mathrm{~K}$. Similar conditions have been found in two out of the four $\mathrm{HH}$ objects we investigated in previous studies, namely HH43 (Paper I) and HH120 (Paper II).

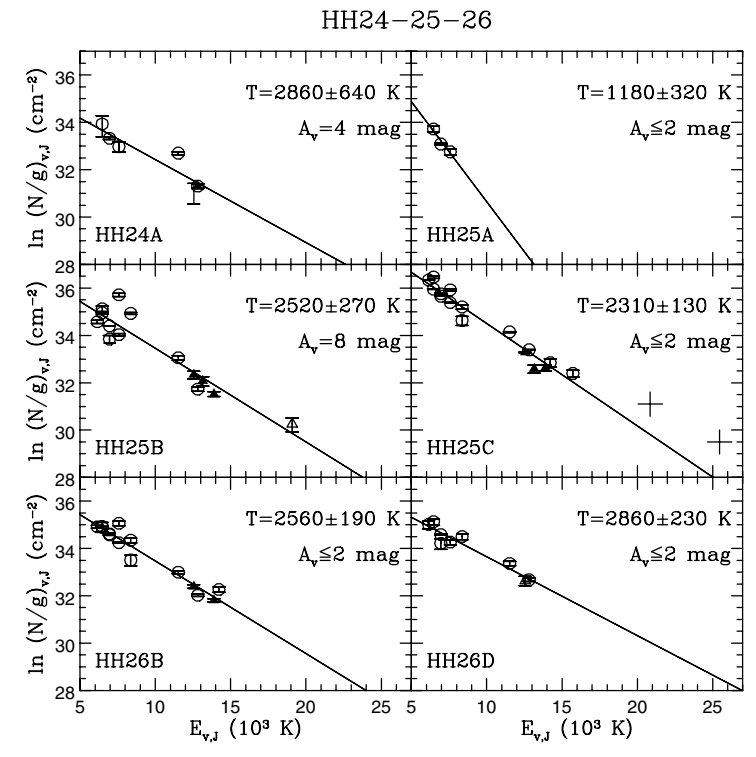

Fig. 9. As in Fig. 8 for the pure $\mathrm{H}_{2}$ knots in the $\mathrm{HH} 24-26$ region. The two crosses in the HH25C panel indicate the positions which would correspond to the 3-1 S(5) and 4-2 S(5) lines by assuming the same ratios with the 1-0 S(1) line as in the HH26C case (see text).

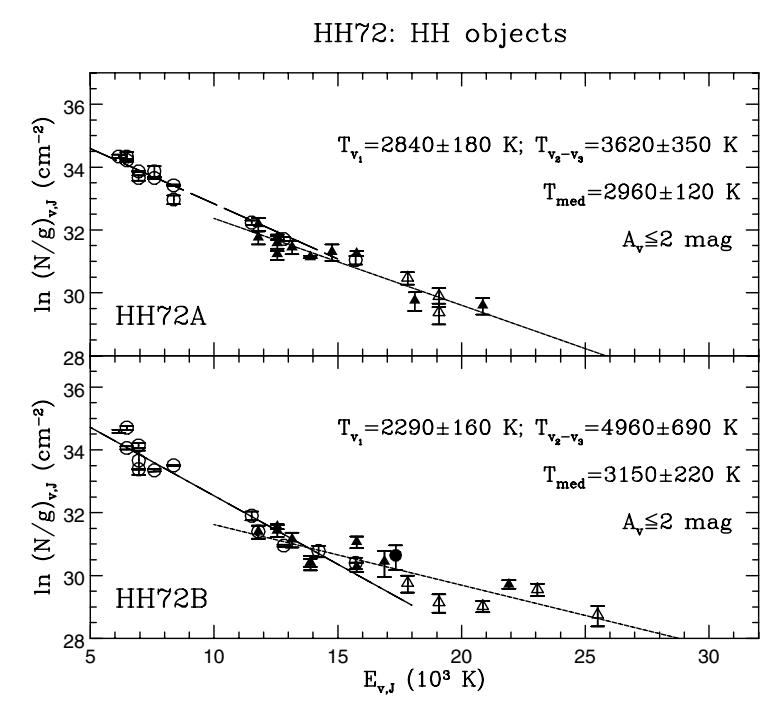

Fig. 10. As in Fig. 8 for $\mathrm{HH} 72 \mathrm{~A}$ and B.

Since the lack of a temperature stratification is deduced only when the highly excited lines (with $v \geq 3$ ) remain undetected, it is crucial to understand whether, in these cases, the observations reflect an intrinsic property of the gas or is attributable to the instrumental detection limit.

In order to investigate this point, we have estimated the fluxes expected for the $v \geq 3$ lines, both from a single temperature gas and in the case of a temperature stratification. As a test case, we considered the spectrum of $\mathrm{HH} 25 \mathrm{C}$, which is a bright knot with a negligible extinction, observed in only $v=1,2$ lines, which can be fitted by a single temperature $\approx 2300 \mathrm{~K}$ (Fig. 9, middle right panel). By extrapolating the straight line up to energies of $\approx 25000 \mathrm{~K}$, we can evaluate the fluxes of the $v=3,4$ lines that would be expected in 
$\mathrm{HH} 72: \mathrm{H}_{2}$ knots

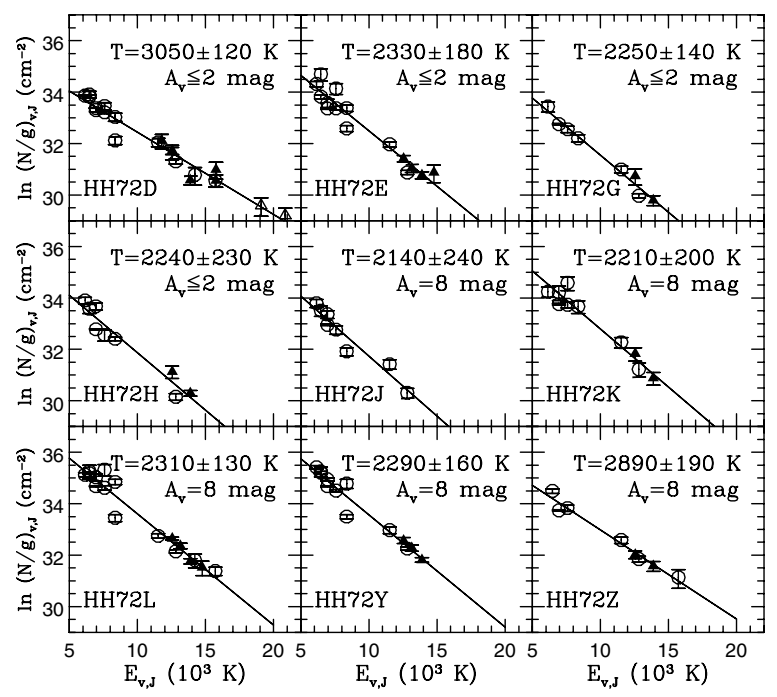

Fig. 11. As in Fig. 8 for the pure $\mathrm{H}_{2}$ knots in the HH72 region.

BHR71: $\mathrm{HH}$ objects

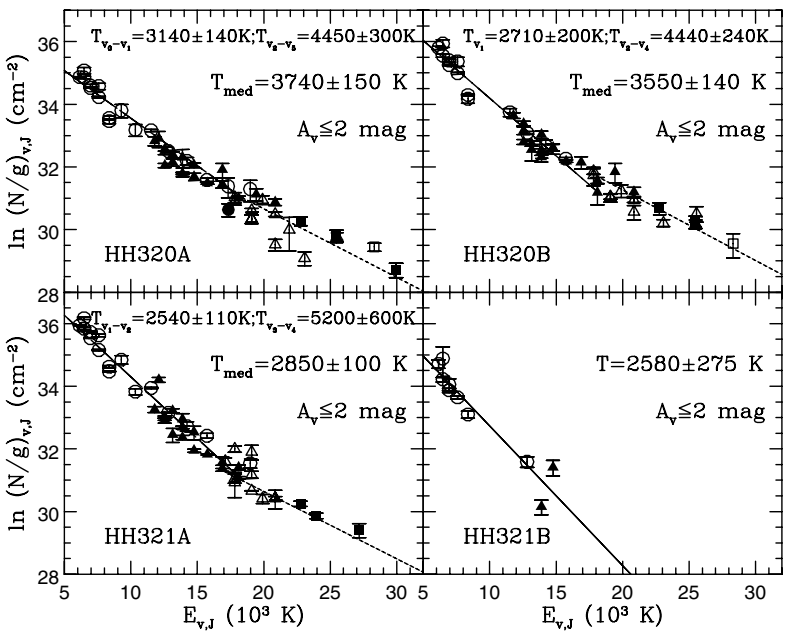

Fig. 12. As in Fig. 8 for HH 320A/B and HH321A/B.

thermal equilibrium: we find that all the $v=3,4$ lines are definitely below the detection limit. We have estimated the fluxes of the same lines, in case of a temperature stratification, by assuming that their ratios with the 1-0 S(1) line are the same as those measured in HH26C (a knot with brightness and extinction comparable to $\mathrm{HH} 25 \mathrm{C}$, but showing a spectrum richer of $v=3,4$ lines). In Fig. 9, the two crosses indicate the corresponding column densities of the 3-1 S(5) and 4-2 S(5) lines: both these points lie above the $3 \sigma$ detection limit. Thus we conclude that the absence of these lines in the spectrum of $\mathrm{HH} 25 \mathrm{C}$ points to excitation conditions different from $\mathrm{HH} 26 \mathrm{C}$.

This analysis has been repeated for other bright knots (such as HH72D and knots 4 and 9 in BHR71), yielding the same conclusion as in the case of $\mathrm{HH} 25 \mathrm{C}$. Thus temperatures rarely exceeding $3000 \mathrm{~K}$ appear to be a proper feature of the pure infrared condensations, while in $\mathrm{HH}$ objects significant temperature gradients occur. This tentative conclusion needs to be confirmed by enlarging the observational database.
BHR71: $\mathrm{H}_{2}$ knots

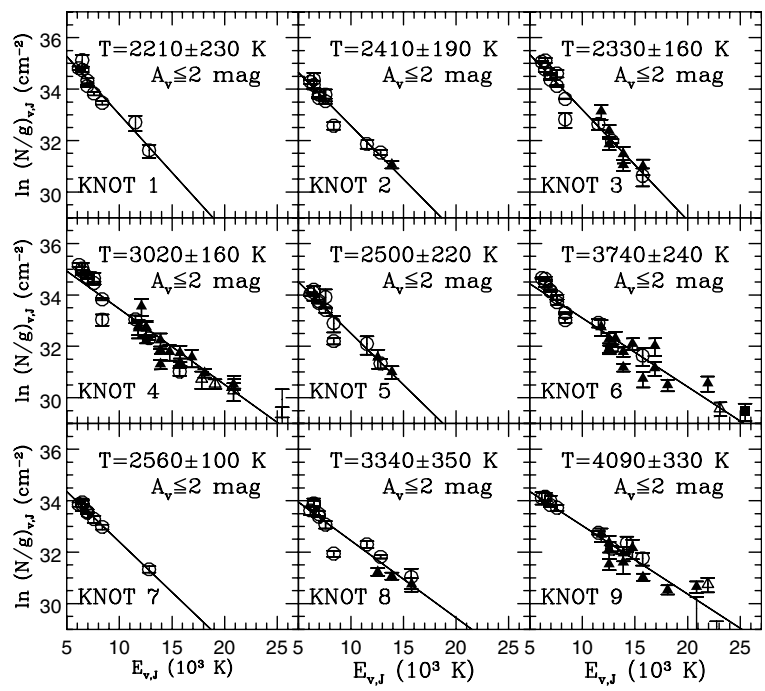

Fig. 13. As in Fig. 8 for the pure $\mathrm{H}_{2}$ knots in the BHR71 region.

\subsection{Atomic and ionic emission}

As we have already pointed out, faint atomic and ionic lines are detected in only four of the objects investigated (HH24A, HH26A and HH72A/B). We first attempt to analyze such emission without making assumptions regarding the underlying excitation mechanism, using simple NLTE models of the equilibrium level populations. We then present the results obtained by modelling the same emission, assuming shock excitation (Sect. 4.4.6).

Following the procedure described in Paper II, we determine the electron density from the [Fe II] $1.644 \mu \mathrm{m} / 1.600 \mu \mathrm{m}$ ratio, obtaining $n_{\mathrm{e}}$ of approximately $5 \times 10^{4} \mathrm{~cm}^{-3}$ for HH24A and $\mathrm{HH} 72 \mathrm{~A}$, a value which can be considered also as a $3 \sigma$ upper limit for HH26A. Since all the transitions of [Fe II] detected come from levels with quite similar excitation energies, their relative intensities are insensitive to the temperature. However, some constraints on the conditions in the ionized gas can be derived by using lines from different species, such as the [S II] and the $[\mathrm{N}$ I] multiplets at $1.03 \mu \mathrm{m}$ and $1.04 \mu \mathrm{m}$. In practice, this can be done for HH72A, the only object where these lines are all detected with a sufficiently high signal-to-noise ratio.

From the [Fe II] $1.644 \mu \mathrm{m} /[\mathrm{S}$ II] $1.03 \mu \mathrm{m}$ ratio we derive the fraction of iron in the gas phase, a parameter regulated in $\mathrm{HH}$ objects by the shock efficiency in destroying the cores of the dust grains in which most of the iron is to be found (cf. May et al. 2000). To this end, we plot, in the upper panel of Fig. 14, the expected [Fe II] $1.644 \mu \mathrm{m} /[\mathrm{S}$ II $] 1.03 \mu \mathrm{m}$ ratio as a function of the temperature, having fixed the electron density at the derived value of $5 \times 10^{4} \mathrm{~cm}^{-3}$. The hatched area refers to the HH72A observations, corrected by $A_{\mathrm{V}}=4.7 \mathrm{mag}$. We assume that all the sulphur is in the gas phase and that both species are singly ionized, as suggested by the absence from the spectra of transitions from any other ionization stage. Then, the plotted ratio is a function only of the temperature and the fraction $f$ of iron in the gas phase. The observational data agree with $f$ values less than 0.4 for all the considered temperatures $(2000<T<15000 \mathrm{~K})$; in particular, if $T=10000 \mathrm{~K}$, we 


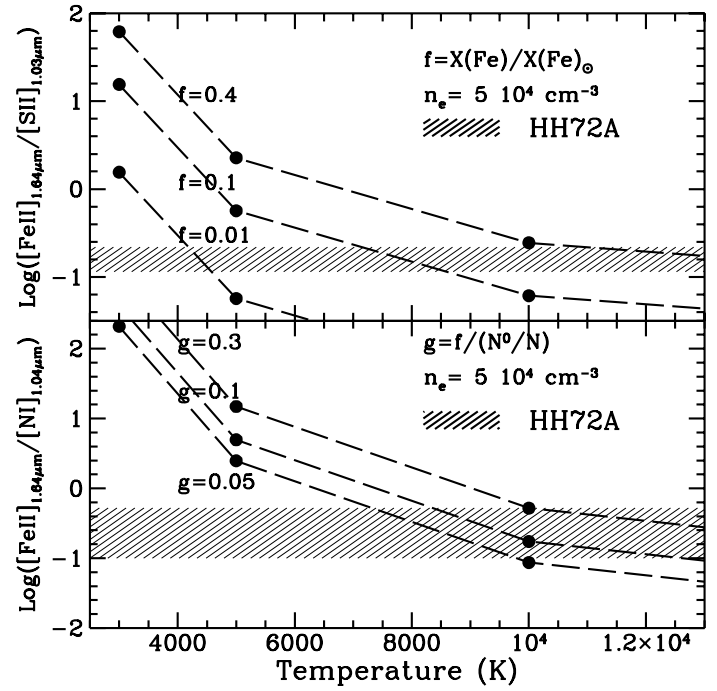

Fig. 14. Top panel: [Fe II] $1.644 \mu \mathrm{m} /[\mathrm{S}$ II $] 1.03 \mu \mathrm{m}$ as function of the temperature, for an electron density of $5 \times 10^{4} \mathrm{~cm}^{-3}$. The curves refer to different assumptions on the iron abundance. The observed ratio in HH72A is drawn as a hatched area. Bottom panel: as in the top panel for the ratio [Fe II] $1.644 \mu \mathrm{m} /[\mathrm{N} \mathrm{I}] 1.04 \mu \mathrm{m}$. The parameter $\mathrm{g}$ is the ratio between the iron abundance and the fraction of neutral nitrogen.

find $0.20<f<0.35$. For comparison, the same analysis applied to HH240A, the object in the survey with the richest iron spectrum, gives $0.6<f<1$, in substantial agreement with the abundance estimated in Paper II by comparing with the hydrogen recombination lines.

Once the iron abundance has been evaluated, it is possible to use the [Fe II] $1.644 \mu \mathrm{m} /[\mathrm{NI}] 1.04 \mu \mathrm{m}$ ratio to estimate the hydrogen ionization fraction $\left(x_{\mathrm{e}}\right)$ and hence the total density of the ionized medium $\left(n_{\mathrm{H}}=n_{\mathrm{e}} / x_{\mathrm{e}}\right)$. The dependence of the above ratio on $x_{\mathrm{e}}$ assumes that nitrogen is at least partially ionized, as is observationally testified by the frequent detection in HH objects of the [N II] optical lines at 6548 and $6583 \AA$ (e.g. Bally \& Reipurth 2001). The considered ratio is plotted in the lower panel of Fig. 14 for different values of the parameter $g$, defined as the ratio between the iron abundance and the fraction of neutral nitrogen.

If we take $T=10000 \mathrm{~K}$, we derive $0.05<g<$ 0.3 , which, for the estimated range of $f$, corresponds to $N^{0} / N>0.67$. Following the procedure described by Bacciotti $\&$ Eislöffel (1999) for deriving the nitrogen ionization equilibrium, $N^{0} / N>0.67$ corresponds to $x_{\mathrm{e}}<0.25$, and to $n_{\mathrm{H}}>2.0 \times 10^{5} \mathrm{~cm}^{-3}$. The same procedure applied to HH240A gives $0.85<x_{\mathrm{e}}<0.95$, in agreement with the quite high excitation/ionization conditions derived for this object.

\subsection{Comparison with predictions of shock models}

In this section, we compare the emission from the observed regions with the predictions of shock models. As representative cases, we have chosen the knots richest in lines: HH26A, HH72A and HH320A. We also model HH25C, which is the "test case", considered in Sect. 4.2 above, of an object which is observed in only $v=1,2$ lines.
The main features and the methods used in the shock model computation are described by Le Bourlot et al. (2002) and Flower et al. (2003). Here, we summarize only those aspects most relevant to the present study. The code, MHD_VODE, simulates one-dimensional, planar, multi-fluid shock waves. It solves the magnetohydrodynamical equations for the three fluids (neutral, positively and negatively charged) in parallel with an extensive chemical network, which links 132 species by 917 reactions.

The manner in which the emission by molecular hydrogen is calculated is also described by Le Bourlot et al. (2002) and Flower et al. (2003). Briefly, the processes which populate and depopulate the rovibrational levels of $\mathrm{H}_{2}$ are: collisional excitation and de-excitation; spontaneous radiative decay; collisional dissociation and ionization; and reformation on grains, which occurs in the wake of the shock wave. The equations for the $\mathrm{H}_{2}$ level populations are solved in parallel with the chemical and dynamical conservation equations. This approach is essential to ensure the accuracy of the computed $\mathrm{H}_{2}$ column densities, because the level populations do not respond instantaneously to changes in the physical state of the gas. Radiative pumping of $\mathrm{H}_{2}$ was not taken into account and is considered to be unimportant, compared with collisional excitation, in the context of the models presented below and for reasons that will be given there.

In the presence of a transverse magnetic field, a "jump" (J-) type shock wave will tend to evolve into a "continuous" (C-) type shock wave in which, prior to the attainment of steady state, a J-type shock remains embedded (Pineau des Forêts et al. 1997; Smith \& Mac Low 1997; Chièze et al. 1998). MHD_VODE can simulate not only steady state J- and C-type shock waves, but also J-type shocks with a magnetic precursor, as well as the equilibrium conditions of the preshock gas (see Flower et al. 2003).

In addition to being observable in rovibrational transitions of $\mathrm{H}_{2}, \mathrm{HH}$ outflows can be traced through the emission of $\mathrm{CO}$ and other molecules and of atoms and ions. In the context of the model, as the temperature and density of the gas increase, $\mathrm{H}_{2}$ is dissociated and $\mathrm{H}$ begins to be ionized. As a consequence, the contribution of radiative cooling by $\mathrm{H}_{2}$ decreases and that of other molecular and atomic species, such as $\mathrm{H}_{2} \mathrm{O}, \mathrm{CO}, \mathrm{O}$, $\mathrm{C}, \mathrm{C}^{+}$and $\mathrm{Fe}^{+}$, assumes greater importance. MHD_VODE incorporates the cooling due to rovibrational transitions of molecules and electronic transitions of atoms, specifically $\mathrm{C}$, $\mathrm{N}, \mathrm{O}, \mathrm{Si}, \mathrm{S}$, and of their ions, as well as $\mathrm{Fe}^{+}$. The strongest ionic/atomic lines observed are those of [Fe II] and [C I]; their predicted intensities are affected strongly by processes within the shock wave. In a C-type shock wave or a magnetic precursor to a J-type shock, the erosion of grains can occur owing to the streaming of charged grains through the neutral gas. At sufficiently high shock speeds, the (refractory) grain cores can be partially eroded, releasing elements such as Fe and $\mathrm{C}$ into the gas phase: see May et al. (2000). Neutral iron is ionized rapidly in the gas phase, predominantly through charge transfer reactions with ions, such as $\mathrm{H}_{3}{ }^{+}$and $\mathrm{H}_{3} \mathrm{O}^{+}$, which have larger ionization potentials. $\mathrm{Fe}^{+}$may then be excited collisionally and emit the $[\mathrm{Fe} \mathrm{II}]$ forbidden line spectrum. The predictions of the model with regard to these two species are considered in 
Table 4. Parameters of shock excitation models.

\begin{tabular}{lcccc}
\hline \hline Object & $v_{\mathrm{s}}$ & $n_{\mathrm{H}}^{a}$ & Age & Radiation field \\
\hline & $\mathrm{km} \mathrm{s}^{-1}$ & $\mathrm{~cm}^{-3}$ & $\mathrm{yr}$ & \\
\hline HH72A & 31 & $10^{4}$ & 185 & Yes \\
HH26A & 52 & $10^{4}$ & 70 & Yes \\
HH320A & 41 & $10^{4}$ & 475 & No \\
HH25C & 45 & $10^{4}$ & $>260$ & Yes \\
HH25C $^{2}$ & 27 & $10^{5}$ & $>680$ & Yes \\
\hline
\end{tabular}

Notes: ${ }^{a} n_{\mathrm{H}}=n(\mathrm{H})+2 n\left(\mathrm{H}_{2}\right)$.

${ }^{1,2}$ Models 1 and 2 of $\mathrm{HH} 25 \mathrm{C}$.

Sect. 4.4.6 below, and information on the atomic data used in the model can be found in the Appendix.

\subsubsection{Model parameters}

It was found that, for HH72A, HH26A and HH320A, neither steady state C-type nor steady state J-type shocks (which are obtained when the magnetic field is sufficiently weak) could satisfactorily reproduce the observations. The former underestimated the column densities of the levels of high excitation, whereas the latter underestimated the column densities of the levels of low excitation. However, non-equilibrium models of J-shocks with magnetic precursors provided good fits to the $\mathrm{H}_{2}$ observations of these three $\mathrm{HH}$ objects. On the other hand, the excitation diagram of $\mathrm{HH} 25 \mathrm{C}$ could be reproduced by a steady state C-type shock model. In this case, the introduction of a $\mathrm{J}$-component resulted in the populations of levels of high excitation being overestimated.

The optimal values of the shock speed and preshock gas density (in the range from $10^{3}$ to $10^{5} \mathrm{~cm}^{-3}$ ) were determined by comparing the predicted $\mathrm{H}_{2}$ excitation diagram with the observations. The relation $B(\mu \mathrm{G})=\left[n_{\mathrm{H}}\left(\mathrm{cm}^{-3}\right)\right]^{0.5}$ was adopted as defining the initial value of the magnetic induction. Two additional parameters are the age of the shock wave, which determines the extent of the magnetic precursor, and the strength of the ultraviolet radiation field. The model parameters that produce optimal fits to the excitation diagrams for each object are presented in Table 4. The entry in the final column indicates whether the effects of an ultraviolet radiation field (via photoionization processes) were included in the model. HH72A, $\mathrm{HH} 26 \mathrm{~A}$ and $\mathrm{HH} 25 \mathrm{C}$ are located in the vicinity of sources of ultraviolet radiation, and, for these objects, we included photoionization processes with rates corresponding to the mean interstellar radiation field in the solar neighbourhood (Draine 1978). On the other hand, there appear to be no sources of ultraviolet radiation nearby $\mathrm{HH} 320 \mathrm{~A}$, and, in this case, photoionization processes were neglected. It is perhaps significant that the inclusion of a radiation field improved the fits to the empirical excitation diagrams for HH72A, HH26A and HH25C but made worse the fit for HH320A.

The rate of photodissociation of $\mathrm{H}_{2}$ by the mean interstellar radiation field is of the order of $10^{-11} \mathrm{~s}^{-1}$ (cf. Abgrall et al. 1992). The same process (of photon absorption) which gives rise to photodissociation also gives rise to $\mathrm{H}_{2}$ fluorescence through radiative cascade from the excited (optically pumped) electronic states to the rovibrational levels of the electronic ground state. Optical pumping followed by fluorescence is an order of magnitude more probable than optical pumping followed by dissociation of $\mathrm{H}_{2}$. Thus, the total rate of the fluorescence cascade is of the order of $10^{-10} \mathrm{~s}^{-1}$. On the other hand, any given rovibrational level of the electronic ground state receives only a fraction of the cascades. We take $10^{-11} \mathrm{~s}^{-1}$ as an order of magnitude estimate of the rate of population of any given excited rovibrational state through the optical pumping by the unshielded mean interstellar radiation field. We shall now compare this rate with that of collisional excitation.

At kinetic temperatures of a few thousand kelvin, attained in the shock wave, the rate coefficients for rovibrational excitation of $\mathrm{H}_{2}$ by $\mathrm{H}$ are typically of the order of $10^{-12} \mathrm{~cm}^{-3} \mathrm{~s}^{-1}$ and exceed those for excitation by $\mathrm{He}$ and $\mathrm{H}_{2}$ by more than a factor of 10 (cf. Le Bourlot et al. 1999). Furthermore, the fractional abundance of $\mathrm{H}$ in the shock wave becomes comparable with that of He, i.e. of the order of 0.1 . In the models considered below, the preshock gas density $n_{\mathrm{H}} \geq 10^{4} \mathrm{~cm}^{-3}$. Thus, the rate of rovibrational excitation of $\mathrm{H}_{2}$ by $\mathrm{H}$ is of the order of $10^{-9} \mathrm{~s}^{-1}$ or greater. We conclude that the inclusion of optical pumping would not have a significant effect on computed column densities of the excited rovibrational levels of $\mathrm{H}_{2}$ and hence on their comparison with the observations.

Low preshock gas densities result in low rates of collisional excitation of $\mathrm{H}_{2}$ molecules, smaller excited state column densities and greater departures of the level populations from LTE. For a given density, the shock speed is the main factor determining the column densities: an increase in the shock speed results in larger column densities. Indeed, the model is so sensitive to the shock speed that a change of the order of $1 \mathrm{~km} \mathrm{~s}^{-1}$ in $v_{\mathrm{s}}$ can make a significant difference to the excitation diagram. The age of the outflow affects differentially the population densities of the more highly excited levels: the younger the object, the closer is the shock wave to being J-type and the higher are the column densities of the more highly excited rovibrational levels. Furthermore, the populations of these levels tend to be closer to LTE. The presence of a radiation field leads to an increase in the degree of ionization of the gas and hence in the strength of the coupling between the neutral and the charged fluids; there results a narrower shock wave.

The observations comprise emission lines from $\mathrm{H}_{2}$ levels of both ortho (J odd) and para (J even) symmetry and hence reflect the variation of the ortho:para ratio through the shock wave. In the hot gas produced by the shock wave, para- $\mathrm{H}_{2}$ is converted to ortho- $\mathrm{H}_{2}$ by nuclear spin changing reactions with $\mathrm{H}$. As shown by Wilgenbus et al. (2000), the local ortho:para ratio increases from a low value in the cold preshock gas (assuming that it has become thermalized through proton transfer reactions with $\mathrm{H}^{+}$and $\mathrm{H}_{3}^{+}$) to a value of 3 immediately behind the shock wave. The ratio of the total column densities in the ortho and para levels, $N$ (ortho)/ $N$ (para), lags behind the local value of the ratio of the number densities of the ortho and para levels. However, our observations do not yield $N$ (ortho)/ $N$ (para), as the contributions from the levels of the vibrational ground state, whose populations become significant in the cooling flow, are not included. Rather, the observations (of excited vibrational levels) reflect the value of the ortho:para ratio in the region of 


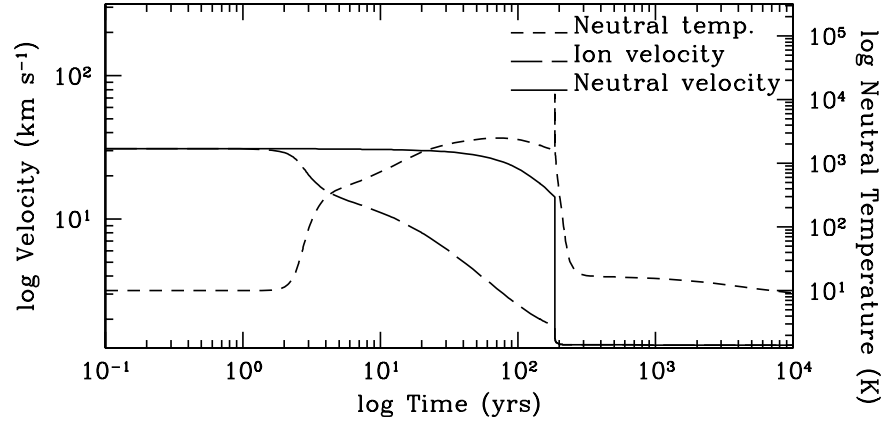

Fig. 15. Velocity and temperature profiles of the model of HH72A. The velocity is expressed in the shock frame and the preshock gas is to the left. The J-shock discontinuity is evident in both the temperature and velocity profiles.

the hot gas in which the corresponding levels are preferentially excited. Thus, we consider that any attempt to derive a unique "ortho:para ratio" from our observations would be misguided. On the other hand, any systematic departure of the ortho:para ratio from its value in LTE would manifest itself as a downwards displacement of the ortho levels in the excitation diagram (cf. Wilgenbus et al. 2000); no such shift is discernible in the comparison with the models presented below.

\subsection{2. $\mathrm{HH} 72 \mathrm{~A}$}

The velocity and temperature profiles of the model for HH72A are plotted against flow time, $t_{n}=\int \frac{1}{v_{n}} \mathrm{~d} z_{n}$, in Fig. 15: the time at which the J-type discontinuity occurs may be identified with the age of the shock wave. The models of HH26A and HH320A exhibit qualitatively similar velocity and temperature profiles, which will not be shown.

In Fig. 16 (top panel) we show the excitation diagrams derived from the model (open symbols) and the observations (corresponding filled symbols). The observations extend up to approximately $2 \times 10^{4} \mathrm{~K}$ above ground and include $v=1,2$ and 3 vibrational levels. At successive vibrational thresholds, the observational points do not depart notably from the median curve, indicating that there is a high degree of thermalization at the local temperature. Models with initial densities less than $10^{4} \mathrm{~cm}^{-3}$ yield lower column densities which diverge more evidently from the median curve at each vibrational threshold. Conversely, models with initial densities higher than $10^{4} \mathrm{~cm}^{-3}$ produce level populations which are closer to LTE, but the curvature of the Boltzmann plot is not consistent with that observed. Similar conclusions were reached from the analysis of the spectra of both HH26A and HH320A.

The characteristic length of the shock wave is determined by the distance over which the ion and neutral velocities differ. For HH72A, this length is approximately $2 \times 10^{16} \mathrm{~cm}$, which may be compared with the observed dimension of the source, $3.5 \times 10^{16} \mathrm{~cm}$ (at a distance of $1500 \mathrm{pc}$ and for an angular size of 1.5 arcsec, as measured on the image taken at the telescope). The same comparisons for HH26A and HH320A produced similar levels of agreement.

\subsection{3. $\mathrm{HH} 26 \mathrm{~A}$}

The observed points in the $\mathrm{H}_{2}$ excitation diagram extend, in this case, to almost $3 \times 10^{4} \mathrm{~K}$ and include levels of the first four excited vibrational states: see Fig. 16, middle panel. The column densities are larger than observed in HH72A, implying a higher shock speed, and show a similar degree of departure from LTE. The higher shock speed used for HH26A results in a higher rate of $\mathrm{H}_{2}$ cooling and so the shock is narrower than $\mathrm{HH} 72 \mathrm{~A}$; it is for this reason that the model of HH26A has a smaller age than HH72A.

The model of HH26A yields column densities of levels with $v=1$ which exceed the observations beyond approximately $10^{4} \mathrm{~K}$ (cf. Fig. 16); this is symptomatic of a more general tendency of the models to overestimate the populations of the levels in the $v=1$ manifold. Such a tendency may point to an overestimation of the rates of vibrationally inelastic $v=0 \rightarrow v=1$ collisions. The model of HH26A has the highest shock speed of the three objects that we consider and hence has the largest fractional abundance of $\mathrm{H}$, owing to collisional dissociation of $\mathrm{H}_{2}$. As the rate coefficients for vibrational excitation of $\mathrm{H}_{2}$ by $\mathrm{H}$ are much larger than for excitation by $\mathrm{He}$ or $\mathrm{H}_{2}$, a larger $\mathrm{H}$ abundance exacerbates the effect of any systematic error in the $\mathrm{H}-\mathrm{H}_{2}$ collisional rate coefficients. However, we stress that there is no independent evidence to confirm the existence of such a systematic error.

\subsection{4. $\mathrm{HH} 320 \mathrm{~A}$}

Levels of the first six vibrational states, with excitation energies extending to over $3 \times 10^{4} \mathrm{~K}$, are observed: see Fig. 16 , bottom panel. The column densities observed for two levels with $v=3(E=20857 \mathrm{~K}$ and $E=23070 \mathrm{~K})$ fall significantly below the mean curve in the excitation diagram. The level at $E=20857 \mathrm{~K}$ has been observed twice, in two different transitions: one observation falls on the median curve, while the other, as already stated, falls below it and out of line with the remaining $v=3$ levels. We believe that this discrepancy arises from systematic errors (related to atmospheric absorption) in the observations and that the misalignment of the observational point at $E=23070 \mathrm{~K}$ level is attributable to a similar effect. The absence of a radiation field in this model results in a wider shock wave than is the case for the other objects and hence to a greater shock age.

No atomic or ionic emission is observed towards the BHR71 region and the absence of both [Fe II] and [C I] lines, which were observed towards all other outflows considered in this paper and in Papers I and II, is particularly surprising; here we discuss why these lines are not observed.

Perhaps the most extreme possibility is that there is very little $\mathrm{Fe}$ and $\mathrm{C}$ present in the gas phase of the BHR71 region because they are mainly in solid (and, in the case of $C$, in molecular) form, which would imply that the dust grains have undergone little erosion. Whilst such a situation cannot be discounted by our observations, we consider that it is unlikely because, according to our modelling results, the gas has been subjected to a strong shock. Emission from [C I] and [Fe II] would not be observed if $\mathrm{C}$ and $\mathrm{Fe}^{+}$were ionized by collisions, 


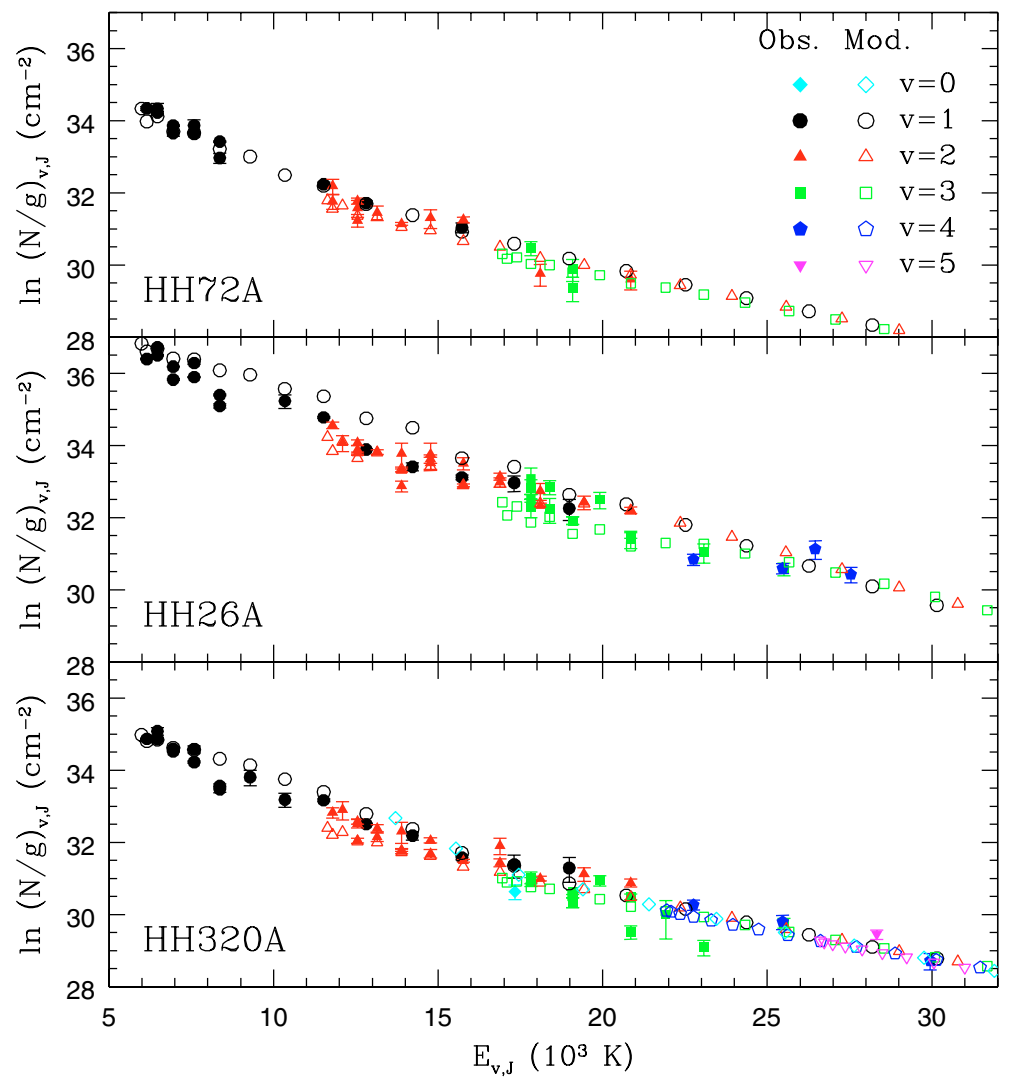

Fig. 16. Models predictions for the excitation diagrams of HH72A, HH26A and HH320A. Different symbols distinguish transitions coming from different manifolds.

photons or charge transfer reactions; but it should be noted that we observe no lines of $\mathrm{Fe}^{2+}$ in this region. The energies required for ionization of $\mathrm{C}$ and $\mathrm{Fe}^{+}(11.26 \mathrm{eV}$ and $16.16 \mathrm{eV}$, respectively) are such that, if collisional or photoionization is invoked, we should expect emission from other ions (for example, [S II] and [N II]) that are not observed. Charge transfer ionization of $\mathrm{C}$ to $\mathrm{C}^{+}$is ineffective and $\mathrm{Fe}^{2+}$ rapidily recombines via charge transfer recombination reactions with $\mathrm{H}$ (Kingdon \& Ferland 1996). For these reasons, we consider that ionization is unlikely to be responsible for the absence of [C I] and [Fe II] emission. Collisional deexcitation of the lines could be responsible for their relative weakness. The rate of electron collisional deexcitation of the [Fe II] and [C I ] lines is of order $10^{-7}$ and $10^{-9} \mathrm{~cm}^{3} \mathrm{~s}^{-1}$, respectively: by comparison, the radiative decay rates are of order $10^{-3} \mathrm{~s}^{-1}$ for [Fe II] and $10^{-4} \mathrm{~s}^{-1}$ for [C I] Therefore, in order for collisional deexcitation to dominate radiative decay, $n_{\mathrm{e}}$ must be greater than $10^{4}$ or $10^{5} \mathrm{~cm}^{-3}$, respectively, which would imply that hydrogen is fully ionized. An alternative and more probable explanation is that the atomic and ionic emission arises in the apex of a bow shock or a reverse shock in the jet. In either case, the region of atomic and ionic emission would be small, and it is possible that the slits did not encompass fully the emitting areas.

\subsection{5. $\mathrm{HH} 25 \mathrm{C}$}

As noted in Sect. 4.2, HH25C is an object which is observed in $v=1,2$ lines, at excitation energies extending up to $\approx 1.5 \times 10^{4} \mathrm{~K}$, beyond which we have only upper limits on the line intensities and the column densities of the corresponding levels.

We present two models of $\mathrm{HH} 25 \mathrm{C}$, whose parameters are given in Table 4. Both the models are of C-type shocks in steady state, and the flow time through the shock wave provides only a lower limit to its age. The models are compared with the observations of HH25C in Fig. 17. Regarding the levels which are definitely observed, with excitation energies less than about $1.5 \times 10^{4} \mathrm{~K}$, the model with the lower density (and higher shock speed) provides perhaps a better fit: the column densities of the $v=1$ levels are overestimated and those of the $v=2$ levels are underestimated to a greater extent by the higher density (lower velocity) model 2 . On the other hand, the predictions of the higher density model are more consistent with the observational upper limits at excitation energies above $1.5 \times 10^{4} \mathrm{~K}$. Straight line fits to the theoretical data yield excitation temperatures of $2770 \mathrm{~K}$ for model 1 and $2130 \mathrm{~K}$ for model 2; the latter result is more consistent with the value of $\approx 2300 \mathrm{~K}$ obtained by fitting to the observational data.

\subsection{6. $[\mathrm{Fe} \mathrm{II}]$ and $[\mathrm{CI}]$ emission lines}

Emission lines of [Fe II] and [C I] were also observed towards $\mathrm{HH} 72 \mathrm{~A}$ and HH26A. For both objects, the intensities predicted by the models (which successfully reproduce the $\mathrm{H}_{2}$ excitation diagrams) are more than 2 orders of magnitude (in the case of 


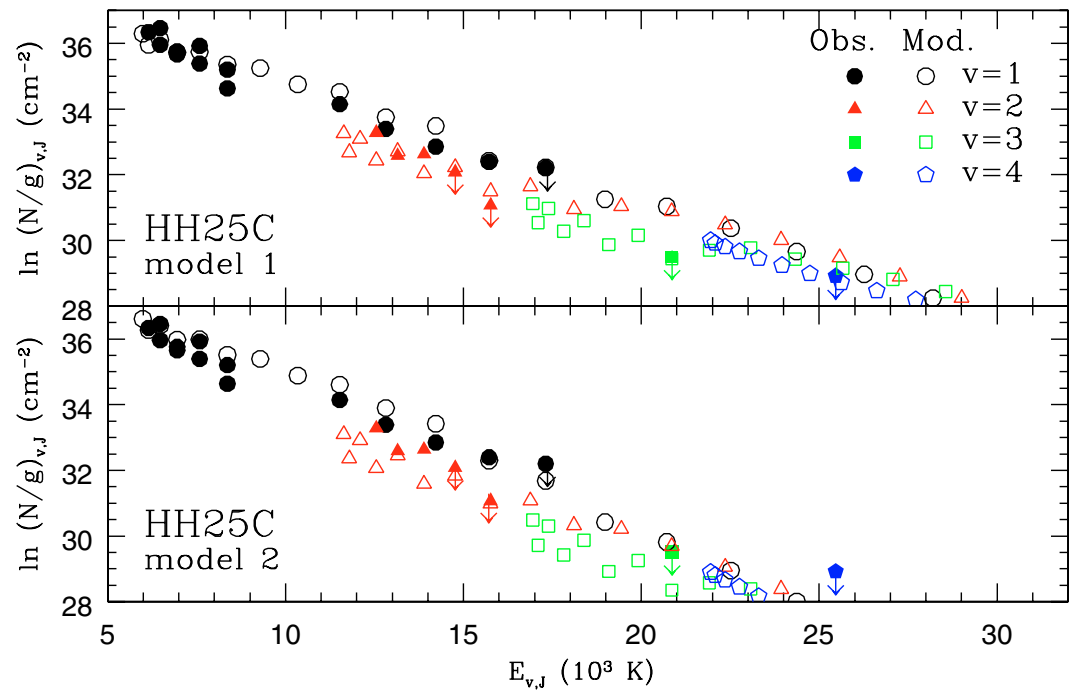

Fig. 17. Excitation diagram of HH25C: the observational data are compared with predictions from two different steady-state C-shock models ( 1 and 2) whose parameters are listed in Table 4 . The arrows indicate $3 \sigma$ upper limits on the column densities of the corresponding transitions.

[Fe II]) and 5 orders of magnitude (in the case of [C I $]$ ) smaller than are observed.

An important difference between the shock wave model and the NLTE model used in Sect. 4.3 is that the shock model predicts a low ionization fraction. For example, the ionization fraction within the shock wave model of HH72A does not exceed about $2 \times 10^{-5}$. This degree of ionization is insufficient for electron collisions to play a significant role. The excitation of $\mathrm{Fe}^{+}$and of $\mathrm{C}$ occurs principally in collisions with neutral perturbers, which are less effective than those with electrons. For the rates of collisional excitation of [Fe II] by electrons to be comparable with the rate of excitation by neutrals, the ratio $x_{\mathrm{e}}=n_{\mathrm{e}} / n_{\mathrm{H}} \approx 10^{-3}$. In order to enhance the computed [Fe II] line intensities to values comparable with those observed requires $x_{\mathrm{e}} \approx 0.1$; this may be compared with the upper limit $x_{\mathrm{e}}<0.25$ derived in Sect. 4.3.

The fact that the models underestimate the [Fe II] and [CI] line intensities suggests that these forbidden lines are emitted from regions where the degrees of dissociation and ionization are much higher, which do not contribute significantly to the $\mathrm{H}_{2}$ line intensities. Such regions could be the apex of a bow shock (with the $\mathrm{H}_{2}$ emission being produced in the wings), a reverse shock in the jet, or photoionized gas. However, the last of these three possibilities would imply local sources of ultraviolet radiation, sufficiently hard and intense to photoionize hydrogen; we know of no observational evidence for the existence of such sources of radiation. Accordingly, we lean towards an explanation in terms of the collisional ionization of hydrogen, in high velocity J-type shocks. Indirect evidence that the [Fe II] and $\mathrm{H}_{2}$ emission lines do, indeed, arise in distinct regions is afforded by the difference in the values of the extinction which are derived from the respective line intensities (at least in the case of HH72A) and from the high values of the electron density derived from the [Fe II] lines. In future studies, we intend to address the issue of the origin the atomic and ionic forbidden lines in a more quantitative manner, within the framework of a shock model.

\section{Conclusions}

We have presented the $0.95-2.5 \mu \mathrm{m}$ spectra of three protostellar jets (HH24-26, HH72, HH320-321). The analysis of the observed features has led to the following conclusions:

- Strong $\mathrm{H}_{2}$ emission lines with excitation energies up to $35000 \mathrm{~K}$ are detected throughout all the Herbig-Haro objects present in the three regions, while infrared knots show lines with excitation energies rarely exceeding $15000 \mathrm{~K}$. This difference in the line emission reflects different excitation regimes: while the condensations observed only in the infrared are excited at a single temperature of $\approx 2000$ $3000 \mathrm{~K}$, Herbig-Haro objects have a temperature stratification, with components up to more than $5000 \mathrm{~K}$, which tend towards the values (of the order of $10^{4} \mathrm{~K}$ ) determined from optical transitions. We note that, in order to trace effectively the highest temperature components, it is essential to obtain observations in the $1.0-1.4 \mu \mathrm{m}$ range, where the lines with high vibrational quantum numbers are located.

- Atomic and ionic emission in form of [Fe II], [S II] and [N I] transitions is detected in only a few $\mathrm{HH}$ objects. The ratios of lines of different species have been used to derive some of the physical parameters of the ionized gas (electron density, iron abundance and ionization fraction), which all indicate that the shocks present in these objects have lower excitation/ionization conditions than in cases where stronger [Fe II] emission occurs.

- The observed $\mathrm{H}_{2}$ emission in the HH objects can be reproduced by models of J-type shocks with magnetic precursors and ages of typically a few hundred years. The shock speeds are in the range from about 30 to about $50 \mathrm{~km} \mathrm{~s}^{-1}$, and the preshock gas density is of the order of $10^{4} \mathrm{~cm}^{-3}$. In the case of the pure $\mathrm{H}_{2}$ knot $\mathrm{HH} 25 \mathrm{C}$, it is possible to fit marginally the observational data by means of a steady state (C-type) shock model with a higher preshock density $\left(10^{5} \mathrm{~cm}^{-3}\right)$. 
- The same planar model that fits the $\mathrm{H}_{2}$ emission systematically underestimates both the [Fe II] and [C I] lines fluxes observed in HH72A and HH26A. We believe that either a bow shock (with atomic and ionic emission originating at the apex of the shock and with $\mathrm{H}_{2}$ arising in the wings) or a reverse shock in denser gas at the centre of the outflow is responsible for the ionic and atomic emission. Supporting evidence comes from the high electron density $\left(\approx 5 \times 10^{4} \mathrm{~cm}^{-3}\right)$ and visual extinction derived from the [Fe II] lines.

Acknowledgements. We thank the anonymous referee for a detailed and constructive report.

\section{Appendix: $\mathrm{Fe}^{+}$and $\mathrm{C}^{0}$ models}

In the model of $\mathrm{Fe}^{+}$, we considered transitions among the 19 energy levels which arise from the $\mathrm{a}^{6} \mathrm{D}, \mathrm{a}^{4} \mathrm{~F}, \mathrm{a}^{4} \mathrm{D}, \mathrm{a}^{4} \mathrm{P}$ and $\mathrm{b}^{4} \mathrm{P}$ terms. All of the observed infrared transitions are emitted from $\mathrm{a}^{4} \mathrm{D}$; the strongest lines are at $1.644 \mu \mathrm{m}\left(\mathrm{a}^{4} \mathrm{D}_{\frac{7}{2}}-\mathrm{a}^{4} \mathrm{~F}_{\frac{9}{2}}\right)$ and $1.257 \mu \mathrm{m}\left(\mathrm{a}^{4} \mathrm{D}_{\frac{7}{2}}-\mathrm{a}^{6} \mathrm{D}_{\frac{9}{2}}\right)$. In order to ensure that the populations of the levels of the $\mathrm{a}^{4} \mathrm{D}$ term, which lies about $1 \mathrm{eV}$ $\left(\approx 10^{4} \mathrm{~K}\right)$ above ground, are converged, we included cascades from the $\mathrm{a}^{4} \mathrm{P}$ and $\mathrm{b}^{4} \mathrm{P}$ terms; the $\mathrm{b}^{4} \mathrm{P}$ triplet lies approximately $3 \mathrm{eV}\left(\approx 3 \times 10^{4} \mathrm{~K}\right)$ above ground.

The rates of collisional and radiative transitions determined the $\mathrm{Fe}^{+}$level populations at each point in the model. The spontaneous radiative transition probabilities computed by Quinet et al. (1996) were adopted. Collisions with both neutral species and electrons were included; for the latter, we used the collision strengths of Zhang \& Pradhan (1995). Regarding the neutral perturbers, we included collisions with the most abundant species, $\mathrm{H}_{2}, \mathrm{H}$ and $\mathrm{He}$. However, we are not aware of any quantum mechanical calculations of cross sections for the electronic excitation of $\mathrm{Fe}^{+}$by these perturbers. Accordingly, we estimated the rate coefficients using a simple classical approximation, which derives from the Langevin "orbiting" model, adopting the polarizability and reduced mass appropriate to each perturber.

Assuming LS coupling (cf. Nussbaumer \& Storey 1980), the conservation of the total electron spin implies that the spin state of $\mathrm{Fe}^{+}$can be changed only by exchange of a bound and an incident electron with oppositely directed spins [if (weak) magnetic interactions are neglected]. Electron collisional excitation of the $\mathrm{a}^{4} \mathrm{D}$ term from the $\mathrm{a}^{6} \mathrm{D}$ ground term proceeds via this electron exchange mechanism. In collisions between $\mathrm{Fe}^{+}$ and $\mathrm{H}$, a similar process of exchange can, in principle, take place, between one of the bound electrons of the ion and the bound electron of the hydrogen atom. In the case of collisions with $\mathrm{He}$, on the other hand, the ground state is a singlet, and exchange with an electron of opposite spin implies a transition to a triplet state. As the lowest energy triplet state of He lies almost $20 \mathrm{eV}$ above ground, such events will be extremely rare under the physical conditions that we are considering. Finally, ground state $\mathrm{H}_{2}$ is also a singlet, and the lowest energy triplet state is repulsive. Hence, transitions to the triplet state lead to dissociation of the molecule and require an energy input of more than $4.5 \mathrm{eV}$. We conclude that transitions involving a change of spin, including excitation of $\mathrm{a}^{4} \mathrm{D}$ from $\mathrm{a}^{6} \mathrm{D}$, are induced at a significant rate only by collisions with electrons and $\mathrm{H}$ atoms.

Collisional and radiative transitions among the five energy levels of neutral $\mathrm{C}$ which arise from the ${ }^{3} \mathrm{P},{ }^{1} \mathrm{D}$ and ${ }^{1} \mathrm{~S}$ terms were incorporated in the model. Following the same reasoning as above, we should include all levels of $\mathrm{C}^{0}$ which contribute significantly, by direct collisional excitation or cascade, to the rates of population of the levels from which transitions are observed. The uppermost ${ }^{1} \mathrm{~S}$ term has an energy of $3.1 \times 10^{4} \mathrm{~K}$, relative to ground. The $0.983 \mu \mathrm{m}$ and $0.985 \mu \mathrm{m}$ lines originate in the ${ }^{1} \mathrm{D}$ term, which has an energy of $1.6 \times 10^{4} \mathrm{~K}$. Population transfer in collisions with $\mathrm{H}$ (Launay \& Roueff 1977), $\mathrm{H}_{2}$ (Schröder et al. 1991), He (Staemmler \& Flower 1991), and $\mathrm{H}^{+}$ (Roueff \& Le Bourlot 1990) was taken into account. The data pertaining to electron collisions and also the radiative transition probabilities were taken from the compilation of Mendoza (1983).

\section{References}

Abgrall, H., Le Bourlot, J., Pineau des Forêts, G., et al. 1992, A\&A, 253,525

André, P., Ward-Thompson, D., \& Barsony, M. 2000, Protostars and Planets IV, ed. V. Mannings, A. P. Boss, \& S. S. Russell (University of Arizona Press), 59

Anthony-Twarog, B. J. 1982, AJ, 87, 1213

Bacciotti, F., \& Eislöffel, J. 1999, A\&A, 342, 717

Bally, J., \& Reipurth, B. 2001, ARA\&A, 39, 403

Benedettini, M., Giannini, T., Nisini, B., et al. 2000, A\&A, 359, 148

Black, J. H., \& van Dishoeck, E. 1987, ApJ, 322, 412

Böhm, K. H., \& Matt, S. 2001, PASP, 113, 158

Bontemps, S., André, P., \& Ward-Thompson, D. 1995, A\&A, 297, 98

Bontemps, S., Ward-Thompson, D., \& André, P. 1996, A\&A, 314, 477

Bourke, T. L. 2001, ApJ, 554, L91

Bourke, T. L., Garay, G., Lehtinen, K. K., et al. 1997, ApJ, 476, 781

Bourke, T. L., Hyland, A. R., \& Robinson, G. 1995, MNRAS, 276, 1052

Carter, B. S., \& Meadow, V. S. 1995, MNRAS, 276, 734

Chièze, J.-P., Pineau des Forêts, G., \& Flower, D. R. 1998, MNRAS, 295,672

Chini, R., Krügel, E., Haslam, C. G. T., et al. 1993, A\&A, 272, L5

Chrysostomou, A., Hobson, J., Davis, C. J., Smith, M. D., \& Berndsen, A. 2000, MNRAS, 314, 229

Corporon, P., \& Reipurth, B. 1997, ed. F. Malbet, \& A. Castets, IAU Symp., 182, 85

Cuby, J. G., Lidman, C., Johnson, R., \& Moutou, C. 2003, ISAAC User Manual

Davis, C. J., Ray, T. P., Eislöffel, J., \& Corcoran, D. 1997, A\&A, 324, 263

Davis, C. J., Stern, L., Ray, T. P., \& Chrysostomou, A. 2002, A\&A, 382,1021

Draine, B. T. 1978, ApJS, 36, 595

Draine, B. T. 1980, ApJ, 241, 1021

Eislöffel, J. 2000, A\&A, 359, 236

Flower, D. R., Le Bourlot, J., Pineau des Forêts, G., \& Cabrit, S. 2003, MNRAS, 341, 70

Garay, G., Köhnenkamp, I., Bourke, T. L., Rodriguez, L. F., \& Lehtinen, K. K. 1998, ApJ, 509, 768 
Giannini, T., Nisini, B., Caratti o Garatti, A., \& Lorenzetti, D. 2002, ApJ, 570, L33 (Paper I)

Giannini, T., Nisini, B., Vitali, F., \& Lorenzetti, D. 2001, A\&A, 379, L17

Gibb, A. G., \& Davis, C. J. 1998, MNRAS, 298, 644

Gibb, A. G., \& Heaton, B. D. 1993, A\&A, 276, 511

Gredel, R. 1994, A\&A, 292, 580

Grevesse, N., \& Sauval, A. J. 1998, Space Sci. Rev., 85, 161

Hakkila, J., Myers, J. M., Stidham, B. J., \& Hartmann, D. H. 1997, AJ, 114, 2043

Hilton, J. L., \& Lahulla, J. F. 1995, A\&AS, 113, 325

Hollenbach, D., \& McKee, C. F. 1989, ApJ, 342,306

Kingdon, J. B., \& Ferland, G. J. 1996, ApJS, 106, 205

Kaufman, M. J., \& Neufeld, D. A. 1996, ApJ, 456, 611

Launay, J. M., \& Roueff, E. 1977, A\&A, 56, 289

Le Bourlot, J., Pineau des Forêts, G., \& Flower, D. R. 1999, MNRAS, 305,802

Le Bourlot, J., Pineau des Forêts, G., Flower, D. R., \& Cabrit, S. 2002, MNRAS, 332, 985

Lemaire, J.-L., Field, D., Pineau des Forêts, G., \& Callejo, G. 2002, Conf. Ser., ed. F. Combes, \& D. Barret (EdP-Sciences), in press

Lidman, C., \& Cuby, J.-G. 1999, SOFI User Manual

May, P. W., Pineau des Forêts, G., Flower, D. R., et al. 2000, MNRAS, 318,809
Mendoza, C. 1983, in Planetary Nebulae, ed. D. R. Flower (Dordrecht: Reidel), IAU Symp., 103, 143

Nisini, B., Caratti o Garatti, A., Giannini, T., \& Lorenzetti, D. 2002, A\&A, 393, 1035 (Paper II)

Nussbaumer, H., \& Storey, P. J. 1980, A\&A, 89, 308

Pineau des Forêts, G., Flower, D. R., \& Chièze, J.-P. 1997, ed. B. Reipurth, \& C. Bertout, IAU Symp., 182, 199

Quinet, P., Le Dourneuf, M., \& Zeippen, C. J. 1996, A\&AS,120, 361

Reipurth, B., Chini, R., Krugel, E., Kreysa, E., \& Sievers, A. 1993, A\&A, 273, 221

Reipurth, B., \& Graham, J. A. 1988, A\&A, 202, 219

Rieke, G. H., \& Lebofsky, M. J. 1985, ApJ, 288, 618

Roueff, E., \& Le Bourlot, J. 1990, A\&A, 236, 515

Schröder, K., Staemmler, V., Smith, M. D., Flower, D. R., \& Jaquet, R. 1991, JPhB, 24, 2487

Schwartz, P. R., Gee, G., \& Huang, Y.-L. 1988, ApJ, 327, 350

Seidensticker, K. J., \& Schimdt-Kaler, T. 1989, A\&A, 225, 192

Smith, M. D. 1995, A\&A, 296, 789

Smith, M. D., \& Mac Low, M.-M. 1997, A\&A, 326, 801

Staemmler, V., \& Flower, D. R. 1991, JPhB, 24, 2343

Wilgenbus, D., Cabrit, S., Pineau des Forêts, G., \& Flower, D. R. 2000, A\&A, 56, 1010

Zhang, H. L., \& Pradhan, A. K. 1995, A\&A, 293, 953 